\title{
Coronal plasma flows and magnetic fields in solar active regions
}

\section{Combined observations from SOHO and NSO/Kitt Peak}

\author{
E. Marsch ${ }^{1}$, T. Wiegelmann ${ }^{1}$, and L. D. Xia ${ }^{1,2}$ \\ 1 Max-Planck-Institut für Sonnensystemforschung, 37191 Katlenburg-Lindau, Germany \\ e-mail: marsch@linmpi .mpg.de \\ 2 also at Armagh Observatory, Armagh BT61 9DG, Northern Ireland, now at University of Science and Technology of China, \\ Hefei, China
}

Received 8 April 2004 / Accepted 27 August 2004

\begin{abstract}
During the early days of the SOHO mission, SUMER observed a few active regions (ARs) connected with sunspots on the Sun and took their images and spectra in various EUV emission lines. In addition to these spectroscopic data magnetograms of the photospheric footpoint regions of the AR loops were available from the MDI on SOHO and the National Solar Observatory/Kitt Peak (NSO/KP), data which here are used to construct the coronal magnetic field of the ARs by force-freefield extrapolation. The combined data set is analysed with respect to the large-scale circulation of coronal matter, which means that the Dopplershifts of various lines used as tracers of the plasma flow are investigated in close connection with the ambient magnetic field, which is found to be either closed or open in the coronal volume considered. The Dopplershift pattern is found to be clearly linked with the field topology, and several regions of strong velocity shear are identified. We also estimate the coronal currents. We discuss the results of this mainly phenomenological correlative study with the perspective to understand coronal heating and mass supply to the extended corona, and with respect to the role played by the field in guiding and constraining plasma flows.
\end{abstract}

Key words. Sun: magnetic fields - Sun: chromosphere - Sun: transition region - Sun: corona - Sun: UV radiation

\section{Introduction}

The photosphere and upper solar atmosphere are known to be structured on all scales by the Sun's magnetic field, which in particular guides and controls the transport of mass, energy and momentum of the coronal plasma. The dynamics of the field as well as its changes in topology involving reconnection are believed to play a key role in the heating and evolution of the corona. A recent review of the observational signatures of reconnection is provided by van Driel-Gesztelyi (2003), and on the small-scale dynamics in active regions (ARs) by de Pontieu (2003). Our present knowledge of the magnetic field in the transition region and beyond largely comes from model extrapolations of the field as measured in the photosphere, respectively chromosphere, into the lower corona.

Modern techniques to compute nonlinear force-free coronal magnetic fields are described by Wiegelmann \& Neukirch (2003). The field in the inner corona (from the surface to a solar radius) determines the field of the extended corona, and thus of the entire heliosphere that is perhaps reaching out to near $100 \mathrm{AU}$. The heliospheric field in three dimensions is reviewed from the Ulysses perspective by Neugebauer (2001). Any quantitative estimates of coronal plasma flows and associated magnetic fields, in the quiet Sun but especially in solar active regions, will help us to understand the supply of mass and heat to the whole corona, and thus its maintenance and expansion in the form of the solar wind.

Imaging and spectroscopy in the far and extreme ultraviolet (EUV) light are the tools allowing one to render field and plasma visible, and to diagnose the plasma state through the analysis of emission lines (by measuring their radiances, widths and Dopplershifts), which originate from the many heavy tracer ions confined in the coronal magnetic field. For a comprehensive modern review of this field see Wilhelm et al. (2004). The EIT (Extreme Ultraviolet Imaging Telescope, Moses et al. 1997) on the SOHO (Solar and Heliospheric Observatory) mission and the imagers on TRACE (Transition Region and Coronal Explorer, see e.g. Aschwanden et al. 2002) have in the past years provided unprecedented views of open and closed coronal structures, such as streamers, prominences, loops and active regions (ARs), and unravelled their complex magnetic fields by imaging the spatial distribution of the tenuous plasma. For a review of this new dynamic and intricate corona see Aschwanden et al. (2001).

Estimates of the coronal plasma density, temperature and velocity have routinely become possible by means of Doppler spectroscopy and line ratio diagnostics of emission lines of multiple ions confined in the coronal magnetic field. These 
techniques are provided onboard SOHO by the spectrometers SUMER (Solar Ultraviolet Measurements of Emitted Radiation, Wilhelm et al. 1997; Lemaire et al. 1997) and CDS (Coronal Diagnostics Spectrometer, Harrison et al. 1997). Such data are particularly valuable if combined with magnetograms, either obtained from space with MDI (Michelson Doppler Imager, Scherrer et al. 1995) or on the ground, e.g. from the Spectromagnetograph at National Solar Observatory/Kitt Peak (NSO/KP). These data enable via field extrapolation, e.g. according to Wiegelmann \& Neukirch (2002), a more complete analysis of the coronal field than just its visualization through the emission of the plasma confined by the field.

It is exactly this combination of field and plasma data which is used here in a comprehensive way to study coronal active regions (ARs) associated with sunspots. The unique virtue of our analysis is the correlative use of measured data, stemming from SUMER, MDI and NSO/KP, together with theoretical data obtained by force-free magnetic field extrapolation after Wiegelmann \& Neukirch (2002). The main goal here is to analyse coronal plasma flows in association with magnetic fields. Three solar active regions will be studied, and their field topologies and coronal plasma flows be scrutinized.

Many spectroscopic observations made in the past years with SOHO and TRACE revealed direct evidence for sizable plasma velocities and multiple-temperature structures in AR loops (for reviews of this broad subject see e.g. Kjeldseth-Moe 2003; Brekke 1998). Steady flows in ARs were recently detected by Winebarger et al. (2002) in observations of coronal EUV loops with TRACE, and apparent intermittent flows above an AR in a bundle of magnetic field loops by Winebarger et al. (2001). Previously, Qiu et al. (1999) reported counter-steaming mass flows in AR loops, made visible as apparent motions in the blue and red wings of $\mathrm{H} \alpha$ emissions, and suggested that these flows occur in filamentary magnetic field structures at the footpoints of the AR loops. They concluded that continuous motion may be essential for maintaining cool and dense material at coronal heights. Possible siphon flows along the magnetic structures (loops) were detected by analysing SUMER data obtained in the active region NOAA 7978B (Spadaro et al. 2000). Teriaca et al. (1999) also found evidence for mass motions and up flows (blue shifts) in an AR.

Photospheric plasma flows in active regions were already observed a long time ago, in particular prior to flares. For example, Yoshimura et al. (1971) and Harvey \& Harvey (1976) reported significant upflows in the photosphere as precursors of solar flares. Observations from Keil et al. (1994) gave evidence that strong horizontal and shearing flows can exist near the flare kernels. Recently, Meunier \& Kosovichev (2003) combined data from SOHO/MDI and the THEMIS solar telescope to analyse photospheric plasma flows and magnetic fields for a flaring active region. These authors observed long-living (several hours) supersonic photospheric plasma flows close to a flaring region. During the flare they observed modifications of the plasma flow and strong permanent changes in the longitudinal magnetic field, but the role of these strong flows and their interaction with the coronal magnetic field is not understood yet.
Here we attempt to throw further light on this complex subject, by studying Doppler shifts of coronal emission lines measured by SUMER in three ARs, however for the first time in close connection with coronal magnetic fields that were constructed by force-free extrapolation from photospheric magnetograms.

\section{Observations of three active regions}

\subsection{Examples of active regions on the Sun}

Two examples of solar sunspots and their associated active regions are first presented in this section to provide an overview of the data and to define the solar context for the following analyses. Figures 1 and 2 present on the left sides full-disk EIT images of the Sun and its corona shining at temperatures between 1.6 and $2.0 \mathrm{MK}$, and on the right sides fullSun photospheric magnetograms. The figures show the quiet Sun after solar minimum, with single large active regions numbered NOAA AR 7981, and the more active Sun approaching the maximum of its activity cycle, with the typical belt of active regions at intermediate latitudes. We will first study the NOAA AR 8534, which is indicated on the image by a small box giving the field of view (FOV) that was scanned by the EUV spectrometer. Similarly, the small frames overlaid on the images of the other ARs give the FOVs of the SUMER instrument.

\subsection{Instrumental aspects and characteristics of the data}

The photospheric magnetograms, which were acquired from the ground during the observations of SUMER, were provided by the NSO/KP and obtained via anonymous ftp transfer of the data, of which a detailed description is given in the work of Jones et al. (1992). The full-disk magnetograms were used. The co-alignement of a NSO/KP magnetogram and a SUMER radiance map was achieved by computing and optimizing the cross correlation between the two images.

The instrument characteristics and the first results of SUMER were in detail described by Wilhelm et al. (1995) and by Wilhelm et al. (1997) and Lemaire et al. (1997). In the present data analysis, we first applied the standard procedures for correcting and calibrating the raw data of SUMER. The procedures included decompression, pixel reversion, as well as flat-field, dead-time, local-gain and geometrical corrections. Two methods were then applied to get the spectralline main parameters (radiance, central position and width). First, we fitted the average profile to a single Gaussian or many Gaussians, thereby using the PIKAIA software (Charbonneau 1995). Second, in order to deduce the Dopplergrams we calculated for every spatial pixel the central position of the line, by integrating the line radiance across a spectral window having the size of four times the full line width at half maximum, and by determining subsequently the location of the $50 \%$ level with sub-pixel accuracy.

This procedure was frequently used before to obtain SUMER Dopplergramms (Dammasch et al. 1999) and dramatically reduced the computing time for a large number of 

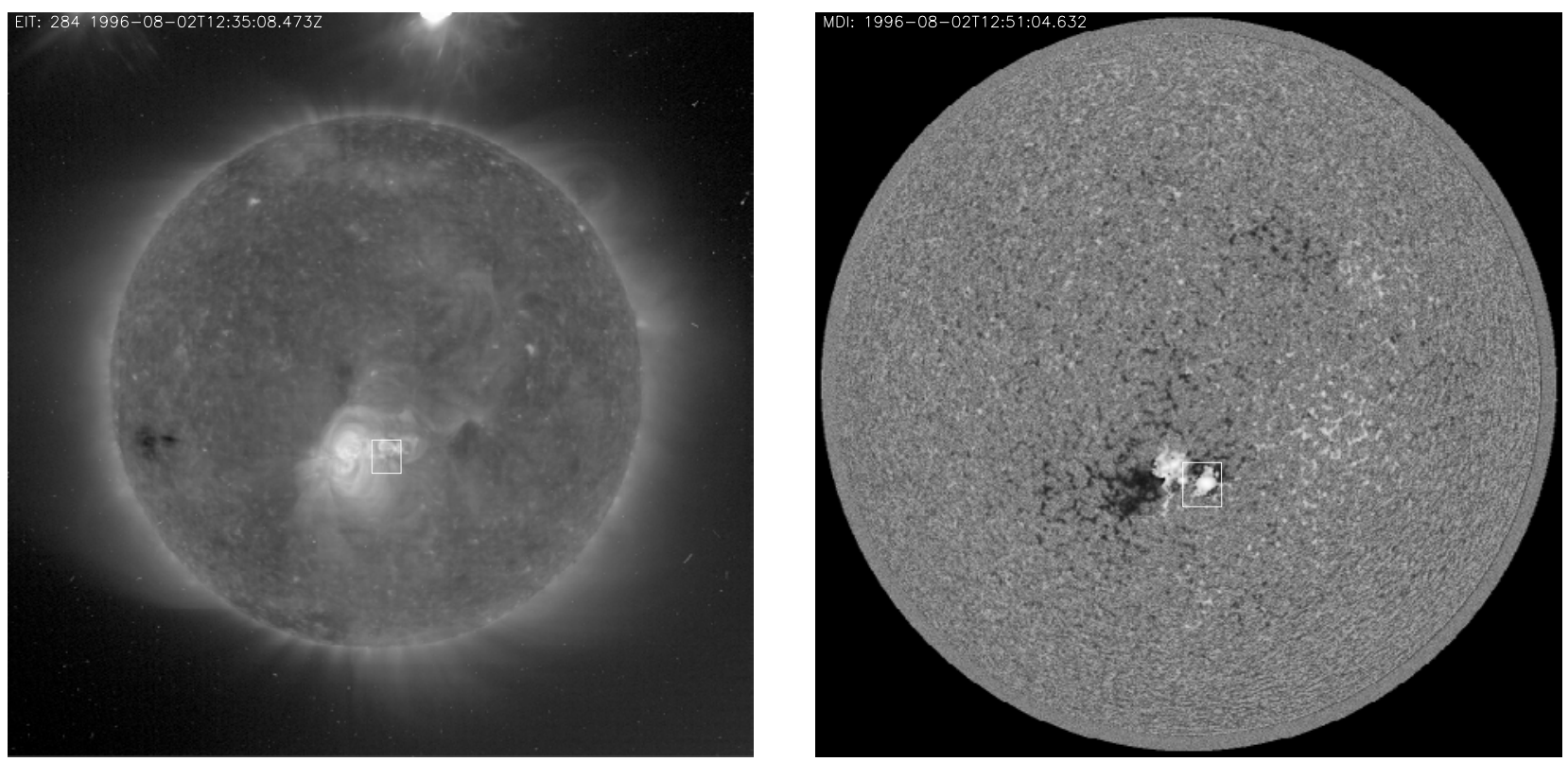

Fig. 1. NOAA AR 7981 in EIT Fe XV $(\lambda 28.4 \mathrm{~nm})$ image (left) observed at 12:35 UTC and MDI magnetogram (right) at 12:51 UTC on 2 August 1996. The small white frames overlaid represent the FOV of SUMER.
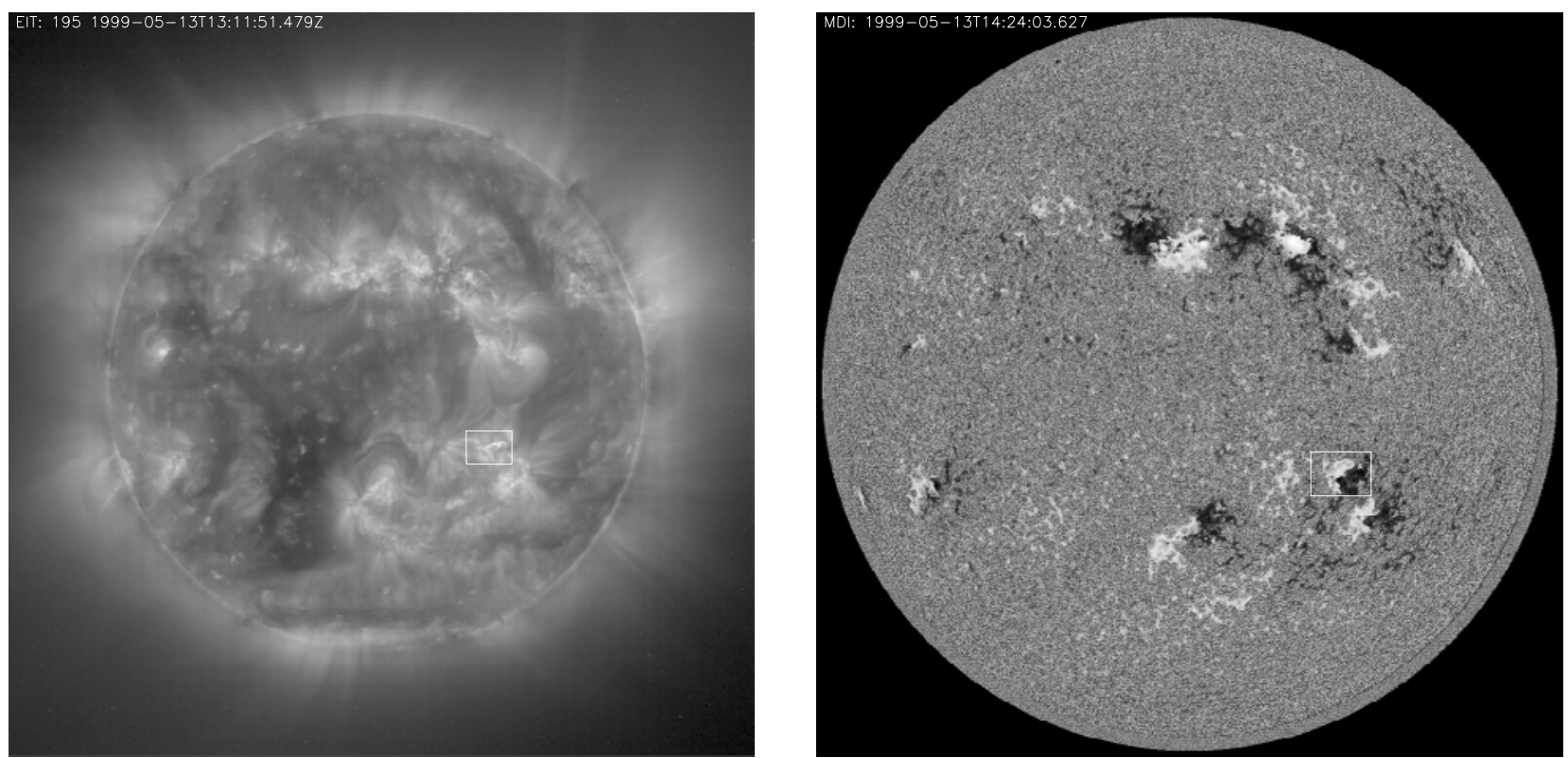

Fig. 2. NOAA AR 8534 in EIT Fe XII ( $\lambda 19.5 \mathrm{~nm}$ ) image (left) observed at 13:12 UTC and MDI magnetogram (right) at 14:24 UTC on 13 May 1999. The small white frames overlaid represent the FOV of SUMER.

data. We found that the results deduced by these two methods were statistically consistent with each other (Xia 2003). Furthermore, it should be mentioned that in addition a lineposition correction was applied, to remove spurious spectral line shifts caused by thermal deformations of the instrument, and to eliminate the residual errors (systematically varying along the slit) which may still remain after the geometric correction using the standard software (Dammasch et al. 1999; Xia 2003).

The Doppler shifts derived from the Ne VIII line are on the average blue on the magnetic field lines that appear to be open to the corona (i.e. to the walls of the field extrapolation box as discussed below). This blueshift seems to be consistent with a field geometry of a coronal fan or funnels, which are open to the corona and where the nascent solar wind is initially accelerated. In previous work (Xia 2003), sizable red shifts were mostly found in the bright structures with mixed-polarity magnetic fields. The Doppler shifts measured for the Ne VIII line in such largely closed fields are generally smaller than in open ones.

\subsection{Past observations and resulting expectations}

Generally speaking, mass may be supplied to (and lost by) the corona either by quasi-steady flows mostly on open field lines and in large loops or in a more transient fashion, e.g. 


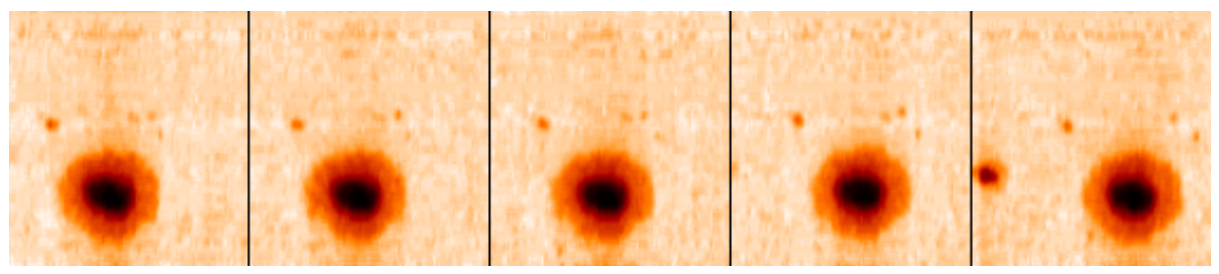

Fig. 3. Sunspot associated with the AR 7981 of 2 August 1996. The SUMER rear-slit camera image in visible light is shown, indicating the large spot and a related group of smaller spots. The time sequence from 11:19 to 14:21 UTC shows the slow evolution of the spot during solar rotation, which is responsible for the displacement of the spot in the FOV.
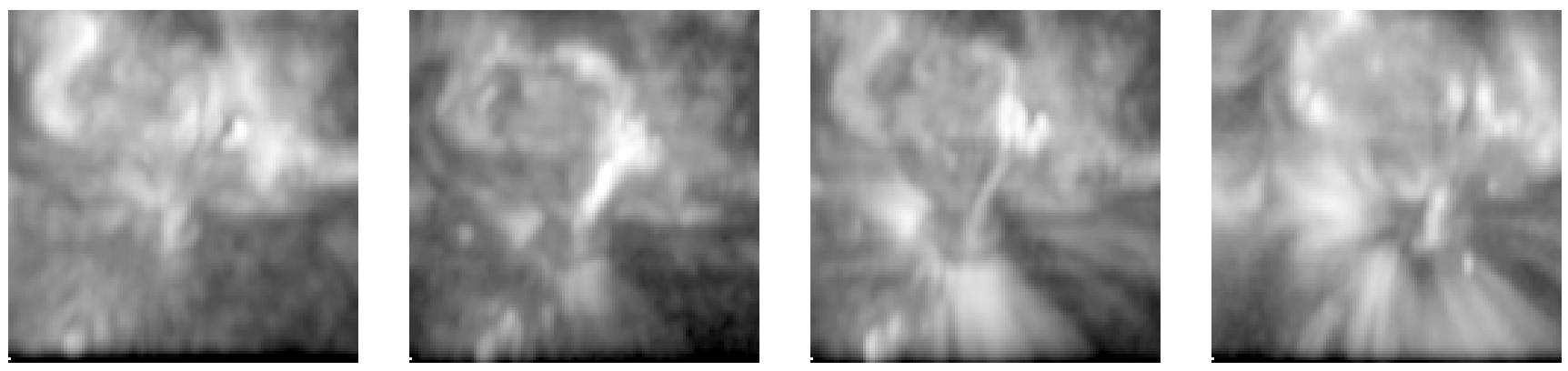

Fig. 4. Sunspot associated with the AR 7981 of 2 August 1996. Solar ultraviolet images of the sunspot in the intensities of the lines (from left to right): H I Ly $\beta(\lambda 102.5 \mathrm{~nm}), \mathrm{N}$ V $(\lambda 123.8 \mathrm{~nm}), \mathrm{O}$ VI $(\lambda 103.1 \mathrm{~nm})$, and finally Ne VIII $(\lambda 77 \mathrm{~nm})$.

through reconnection driven by freshly emerging magnetic flux. The contributions expected theoretically from SOHO a decade ago were reviewed by Antonucci (1994) in the proceedings of the 2nd SOHO Workshop. Bulk motions associated with steady siphon flows in quiet loops, gentle motions in prominences, rapid chromospheric evaporations or coronal condensations, fast outflows from reconnection regions and localized plasma jets, slow coronal rain and draining flows emptying active loops, all these processes possibly contribute to the overall mass budget of the corona. In the meantime (i.e. during the past SOHO/TRACE decade) the observational literature on these types of flows vastly grew, and therefore we will not even attempt to review it here but immediately focus on our three active regions. They are discussed below and found to reveal a mixture of upflows and downflows at several locations above and around the related sunspot.

Obviously, mass can be supplied to and circulated in the corona in various ways defined by the chromospheric plasma sources, the magnetic network and coronal fields guiding the flows, and some plasma is ultimately lost to the solar wind. With SOHO, flows in the lower corona have been routinely measured, e.g. in the magnetic network and all kinds of related structures like small bi-polar loops and multi-polar loop systems. The quiet-Sun transition region (TR) lines forming near $10^{5} \mathrm{~K}$ usually show both red and blue shifts and indicate a complex spatial arrangement of the flows at all scales down to arcseconds. For modern reviews of this subject see for instant the papers of Peter (2001) and Kjeldseth-Moe (2003) and the literature cited therein. The ubiquitous downflows observed in the TR remain a puzzle, however even more so the origin of the mass supply against gravity to the extended corona, which is also necessary to maintain the solar wind mass flux. Above active regions associated with sunspots a particularly rich spatial flow pattern is commonly observed, as it is shown below.

\subsection{Radiance images and Dopplermaps of active regions}

In this section detailed information on the plasma distribution and flow in the two active regions AR 7981 and AR 8534 is provided, for which the global solar context was given in the Figs. 1 and 2. First the sunspot associated with the AR 7981 of 2 August 1996 is shown. These images shown in Fig. 3 were obtained by the SUMER rear-slit camera in visible light. For a description of the camera and its functions see Wilhelm et al. (1995). The time sequence presents the large spot itself and a related group of much smaller spots, and lasts from 11:19 to 14:21 UTC. The slow evolution of the spot during three hours (the displacement is due to solar rotation) is shown. There are no apparent or significant changes in this quiet spot. Note that the direct and extended neighbourhood of the spot is rather uniform and just reveals the common granulation pattern. Nothing indicates any structure, in striking contrast to what the subsequent images taken in EUV light will reveal.

The AR 7981 is shown in Fig. 4 as ultraviolet images obtained in the lines indicated in the figure caption. Note the characteristic appearance of the neon emission which surrounds the spot (that is dark) as a diffuse fanning-out halo. One has the impression as if the fan emission also comes from greater heights and overarches the dark background, which is again consistent with the reduced intensity in the cooler lines. The Lyman line emission (leftist frame) is brighter in the upper two thirds of the figure (and so the other emissions), perhaps indicating denser material trapped there in loops which yield higher emissivities. In particular the bright $\mathrm{C}$-shaped feature in the upper left corner 

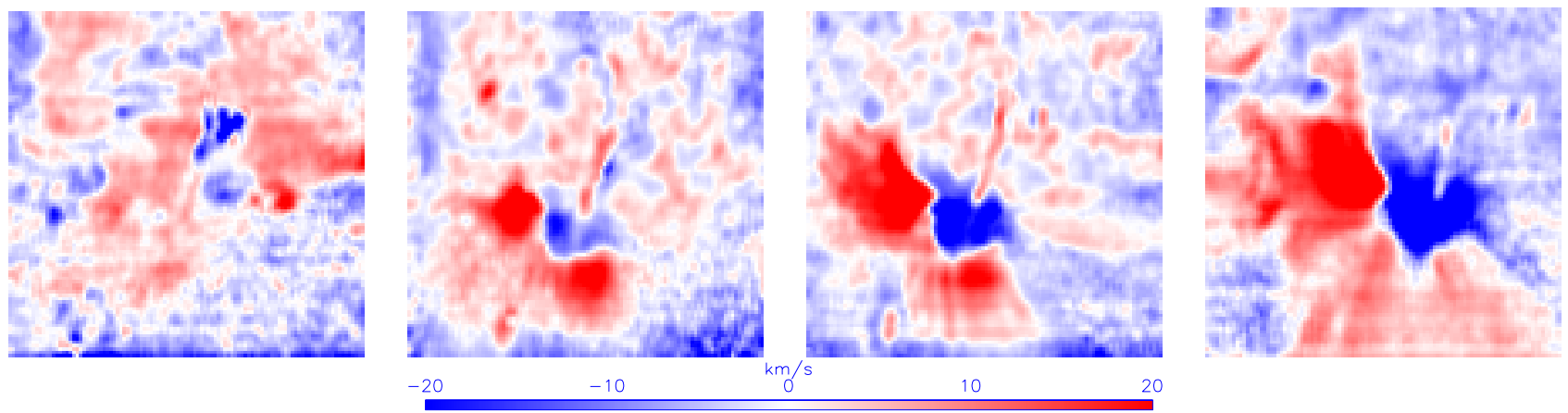

Fig. 5. Sunspot associated with the AR 7981 of 2 August 1996. Solar ultraviolet Doppler images of the sunspot in the shifts of the lines (from left to right): $\mathrm{HI} \operatorname{Ly} \beta(\lambda 102.5 \mathrm{~nm}), \mathrm{NV}(\lambda 123.8 \mathrm{~nm}), \mathrm{O}$ VI $(\lambda 103.1 \mathrm{~nm})$, and finally Ne VIII $(\lambda 77 \mathrm{~nm})$. Note the sharp red-blue boundaries indicating a change in topology from open to closed of the magnetic field confining the plasma and guiding its flow. The red "needle" pointing into the spot indicates plasma downflow, perhaps guided along a magnetic flux tube ending in the spot.

Table 1. Data and SUMER parameters relevant to the SOHO and NSO/KP observations of three solar active regions.

\begin{tabular}{cccccccc}
\hline \hline Item & Date & $\begin{array}{c}\text { Time } \\
\text { UTC }\end{array}$ & $\begin{array}{c}\text { Solar coordinate } \\
\text { X }\left({ }^{\prime \prime}\right), \text { Y }\left({ }^{\prime \prime}\right)\end{array}$ & $\begin{array}{c}\text { Wavelengths } \\
(\mathrm{nm})\end{array}$ & $\begin{array}{c}\text { Steps } \\
\left({ }^{\prime \prime}\right)\end{array}$ & $\begin{array}{c}\text { Detector } \\
\text { and Slit }^{a}\end{array}$ & $\begin{array}{c}\text { Exposure } \\
\text { time }^{(\mathrm{s})}\end{array}$ \\
\hline AR 7953 & 23 March 96 & $19: 49-21: 35$ & $69 \mathrm{E}-268 \mathrm{~W}, 160 \mathrm{~N}-460 \mathrm{~N}$ & $154.0-157.5$ & 1.13 & A 2 & 21 \\
AR 7981 & 2 August 96 & $11: 19-14: 21$ & $13 \mathrm{E}-105 \mathrm{~W}, 333 \mathrm{~S}-213 \mathrm{~S}$ & $102.4-155.0$ & 1.13 & A 6 & $10-50$ \\
AR 8534 & 13 May 99 & $13: 01-14: 49$ & $335 \mathrm{~W}-492 \mathrm{~W}, 302 \mathrm{~S}-182 \mathrm{~S}$ & $152.5-155.5$ & 1.5 & A 4 & 30 \\
\hline
\end{tabular}

${ }^{a}$ Slit 2: $1^{\prime \prime} \times 300^{\prime \prime}$; Slit 4: 1" $\times 120^{\prime \prime}$; Slit 6: 0.3" $\times 120^{\prime \prime}$.

looks like a thick magnetic flux tube. It is apparent in all three adjacent (to the right) frames as well, despite the increasing formation temperature of the EUV emission lines.

Similarly and even more clearly, the same dichotomy of the images is found in the Dopplergrams displayed in Fig. 5. All frames reveal distinct blue shifts in their lower third corresponding to a reduced intensity in the radiance images. The sunspot itself dramatically appears in deep blue in the shifts of the transition region lines of nitrogen, oxygen and neon, which all form above $10^{5} \mathrm{~K}$. Note the sharp boundaries between blue and red, a typical feature which marks the change in magnetic field topology from open to closed, an interpretation that is corroborated subsequently when discussing the magnetic field geometry of another sunspot with its AR.

The bright "needle" pointing into the middle of the spot likely indicates denser plasma on a magnetic bridge, perhaps representing a thick flux tube arching into the spot. Apparently, there are localized niches confining neon that more strongly emits (due to either higher abundance or larger contribution function). These hot blobs or spots are obvious in the upper right corner of the frame via the red shifts in contrast to the ambient blue shifts. These features seem to relate to smaller denser loops with material raining back to the surface, whereas the surrounding emission seems to come from outflowing dilute plasma. Similar finest structures were found in open equatorial coronal holes (Xia 2003).

The fine blue structure in the middle of the $\mathrm{Ly} \beta$ Dopplergram is puzzling. It has no counterpart in the other frames and may relate to up-welling material in small, freshly emerging magnetic flux tubes or upflows in steady loops connected with the sunspot penumbra. Although wavelength calibration is an issue here, the net blue-red contrast would qualitatively not be affected by it, and thus the observed gradients in the shift are real and reliable.

The sunspot associated with the emerging AR 8534 of 13 May 1999 is shown in Figs. 6 and 7. A sequence of solar ultraviolet images of the sunspot region in the intensities and Doppler shifts of the lines from silicon, carbon and neon is given, ordered according to increasing formation temperature. Note the strong structuring of the entire region, indicating a complex underlying magnetic field topology. Note that in this sequence the Sun was first scanned from east to west (between 13:01 and 13:55 UT) and then from west to east (between 13:55 and 14:49 UT). The offset of the field of view is due to solar rotation. This AR was studied before in detail by Winebarger et al. (2002), who presented the first co-aligned SUMER-TRACE observations and detected steady flows in the Ne VIII line, a result which was interpreted as being possibly caused by an asymmetric heating function of the loop.

This double-scanning was done with the intention to detect possible temporal changes in the sunspot area. However, a detailed comparison of the two scans (each lasting about an hour) in Fig. 6 does not reveal any substantial changes in the overall radiance pattern at large scales. But on small scales variations do occur. In particular, in the hotter carbon and neon lines some variable small-scale brightenings occur in the second scan, which did not exist in the first scan. For example, there are several points appearing in all three lines which were bright in the first and fade away or disappear in the second scan, indicating brightness variability on hourly scales or small-scale plasma dynamics.

Also, many thinner threads of plasma (thin magnetic flux tubes) seem to come and go on hourly scales or vary in brightness, in particular the fan-shaped bundle of field lines in the 

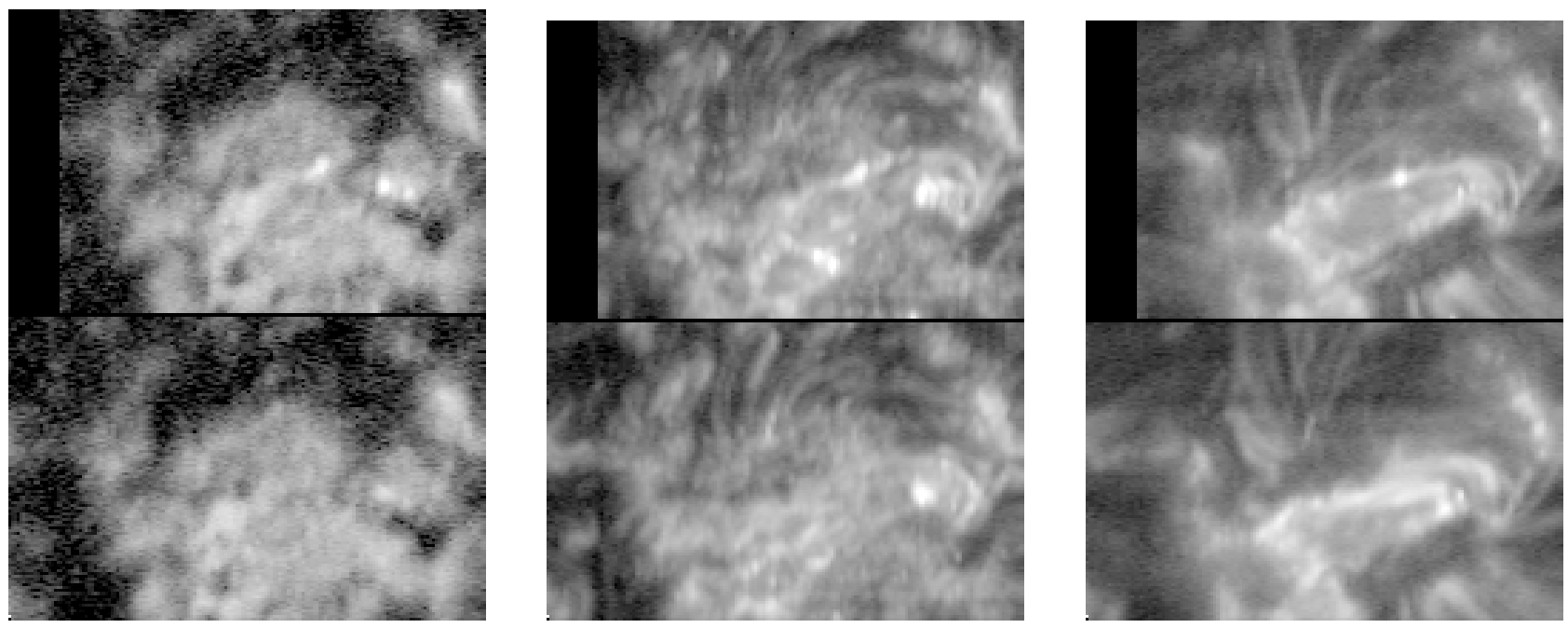

Fig. 6. Solar ultraviolet images of the emerging AR 8534 of 13 May 1999, which is associated with a sunspot. The images were taken in the radiances of the lines (from left to right) of Si II $(\lambda 153.3 \mathrm{~nm})$, C IV $(\lambda 154.8 \mathrm{~nm})$, and Ne VIII $(\lambda 77 \mathrm{~nm})$. Note the strong structuring of the region indicating a complex field geometry including loops and fans. The Sun was scanned from east to west between 13:01 and 13:55 UT with the nominal step minus solar rotation (top panels), and then from west to east between 13:55 and 14:49 UT with the nominal step plus solar rotation (bottom panels). Each scan took $54 \mathrm{~min}$. The offset of the field of view in the top frames is due to solar rotation.
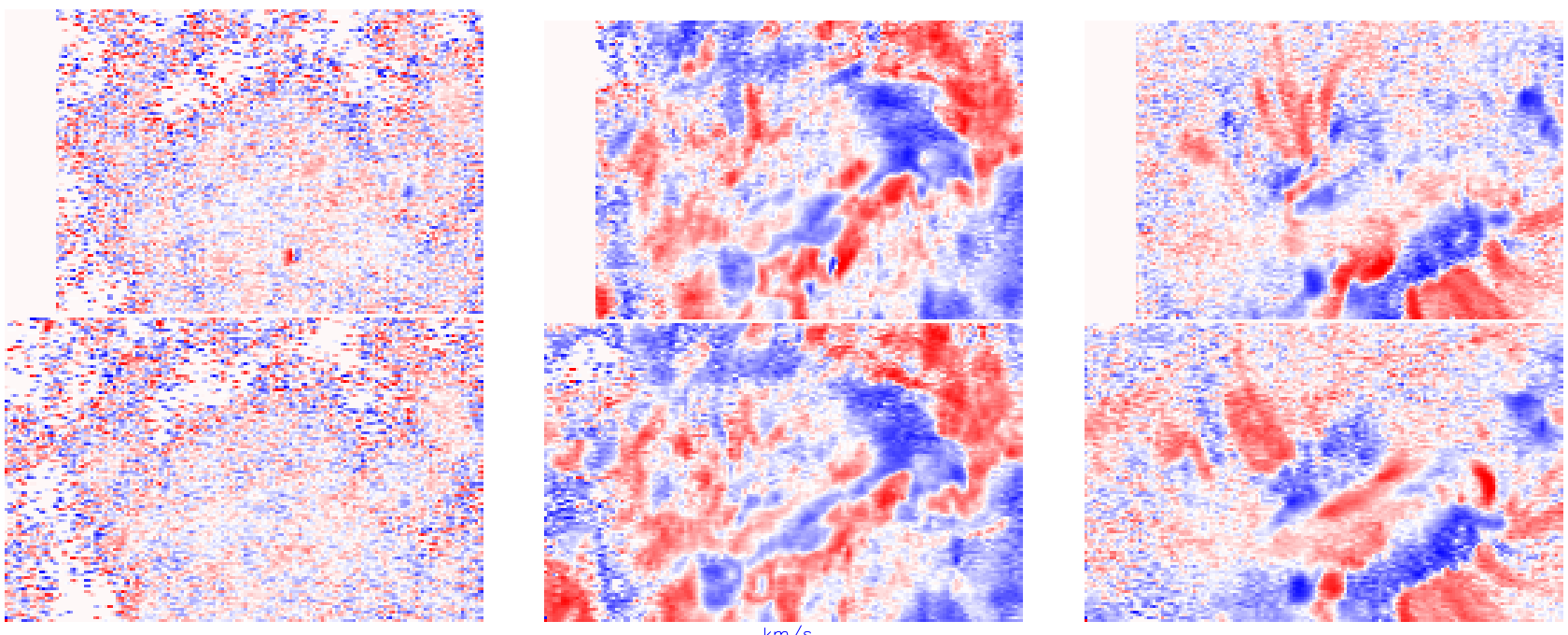

Fig. 7. Solar ultraviolet Dopplergrams of the emerging AR 8534 of 13 May 1999 in the line shifts of the lines (from left to right) of Si II $(\lambda 153.3 \mathrm{~nm})$, C IV $(\lambda 154.8 \mathrm{~nm})$, and Ne VIII $(\lambda 77 \mathrm{~nm})$. Note the varying and sharp red-blue boundaries, indicating changes in field topology on scales much smaller than the whole sunspot area. The Sun was scanned from east to west between 13:01 and 13:55 UT with the nominal step minus solar rotation (top panels), and then from west to east between 13:55 and 14:49 UT with the nominal step plus solar rotation (bottom panels). Each scan took $54 \mathrm{~min}$. The offset of the field of view in the top frames is due to solar rotation.

middle upper part of the frame. Finally, note the clear change in the overall intensity pattern, from diffuse (cold $\mathrm{Si}$ ) over highercontrast (warmer C) to strongly structured (hot $\mathrm{Ne}$ ), which reflects the height dependence of the emission and the magnetic field structure, with individual flux tubes and magnetic arches and filaments which are most conspicuous in the neon emission.

A comparison of the scans (each lasting about an hour) in Fig. 7 does not indicate substantial changes in the overall Dopplershift pattern on large, however on small scales. In particular, the neon line reveals some occurrences of freshly appearing, localized red shifts which did not exist in the previous scan. The sausage-like tube on the right edge of the blue area is at rest in the upper and clearly redshifted in the lower frame an hour later, indicating transient downflows in a loop. Similarly, the fan structure originating in the upper edge in the middle of the large blue speckle in the neon Dopplergram shows spatially and temporally (frame one to two) varying redshifts, likely indicating transient downflows along long flux tubes having small transverse diameters. Flows can exist by various reasons. Winebarger et al. (2002) studied for this AR the implications of an asymmetric heating function and concluded that its consideration improved the agreement between observations and theory. 

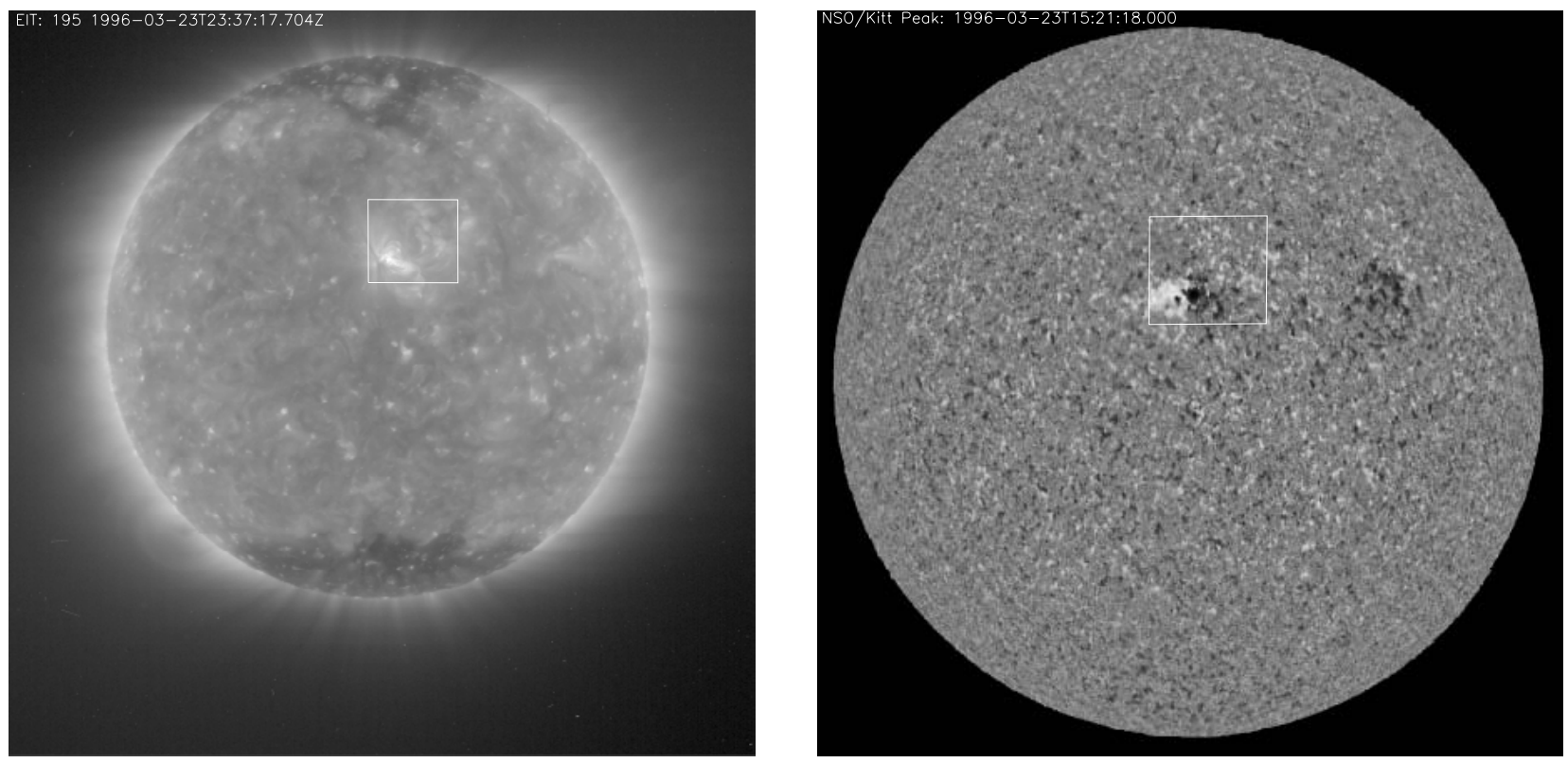

Fig. 8. NOAA AR 7953 in EIT Fe XII ( $\lambda 19.5 \mathrm{~nm}$ ) image (left) observed at 23:37 UTC and NSO/KP magnetogram (right) between 14:54 and 15:48 UTC on 23 March 1996. The small white frames overlaid represent the FOV of SUMER.

When comparing the three Dopplergrams with each other, one finds striking similarities and enigmatic differences between them. For example, the Si Dopplermap overall is weakly structured and shows washed out red-blue patterns, yet there are some larger bluish regions which still have counterparts in the $\mathrm{C}$ but none in the Ne Dopplermaps. Vice versa, in the upper right corner in which a pair of large uniform regions with blue and red shifts is obvious in the frame of the TR C line, the coronal Ne line does not show any Dopplershifts. Also, the large blue patch in the right lower corner of $\mathrm{Ne}$ map is white in $\mathrm{C}$, and the blue patch in $\mathrm{C}$ corresponds to red regions in $\mathrm{Ne}$, indicating opposite plasma flows, which presumably occur at different heights. All these differences are puzzling since they have some, yet not always clear, equivalent differences in the radiance maps of Fig. 6, where similar features can generally be recognized.

These observations illustrate how important it is to have a clue about the background magnetic field, which determines the plasma distribution with height in the corona as well as the flow orientation. However, the observed Dopplergrams alone do not allow conclusions about the origin of the flows, which might be caused by a variety of dynamical processes. Certainly, the structuring of the Dopplermaps hints to complicated smallscale dynamics in association with the nonuniform magnetic field. It is not our intention to speculate further about the causes of the red and blue shifts, but instead to analyse them in considerable detail together with the field for one exemplary active region.

\section{Detailed study of the magnetic structure of AR 7953}

\subsection{EIT and SUMER observations of the active region}

Here we will study the AR 7953 which is presented in its full solar context in the images of Fig. 8, which shows on the left side a full-disk EIT image of the Sun and its corona shining at a temperature of 1.6 MK, and on the right side a full-Sun photospheric magnetogram. We analyse this AR in much more detail than the previous two cases, in particular its associated magnetic field will be reconstructed in order to fully describe and comprehend the coronal plasma environment. The problems encountered in the previous section in the interpretation of the radiance and Doppler maps of EUV emission lines made it clear that knowledge of the field is indispensable when one tries to resolve the observational ambiguities. Ideally, one would like to have simultaneous magnetic field measurements in the corona at the respective locale of the EUV emission. However, a subsequent field extrapolation is the best one can presently do. The required techniques are fully described in a separate section below. First we return to the relevant SOHO/SUMER observations.

\subsection{Magnetic field lines in a complex active region}

The image section given by the white squares in Fig. 8 are now used to construct the three-dimensional coronal magnetic fields in a box of about the size of the FOV of SUMER in that figure. Corresponding enlargements of the FOV in the EIT image presented above will together be shown with the related NSO/KP Peak magnetogram data from 23 March 1996 (14:54-15:48). From the full disk magnetogram (with $1788 \times 1788$ spatial pixels) we extracted a corresponding reduced area consisting of about 50000 pixels with total numbers: $n_{x}=251, n_{y}=201$; running in $x$ from 750 to 1000 and in $y$ from 1000 to 1200 . This area refers on the Sun to a region of length: $L_{x}=287^{\prime \prime} \approx$ $205 \mathrm{Mm}$, and $L_{y}=230^{\prime \prime} \approx 164 \mathrm{Mm}$. The extrapolated magnetic field is illustrated in Fig. 9 by the help of a few typical field lines which are chosen such as to signify the topology of the field and to enable a comparison be made of its flux tubes 
with the EUV emission pattern which visualizes the plasma distribution.

For the purpose of coronal field extrapolation (which is fully explained in the subsequent section), we computed a linear force-free magnetic field with the parameter $\alpha=-1.1 \times$ $10^{-8} \mathrm{~m}^{-1}\left(\alpha L=-2.0\right.$, where $L=254^{\prime \prime} \approx 181 \mathrm{Mm}$ is the harmonic mean of $L_{x}$ and $L_{y}$ ). We used a Fourier representation method for the field calculation (see the subsequent Sect. 3.3.2) and cut off the Fourier series at $m_{\max }=n_{x} / 4=62$ and $n_{\max }=n_{y} / 4=50$. The magnetic field was computed in a box up to a height of 50 pixels $\left(L_{z}=57^{\prime \prime} \approx 41 \mathrm{Mm}\right)$. The $\alpha$-value obtained here is in nice agreement with the ones obtained in a previous comprehensive investigation of more than hundred ARs by Pevtsov et al. (1997), who found from groundbased vector magnetograms and Yohkoh X-ray observations typical values of $\alpha= \pm 2 \times 10^{-8} \mathrm{~m}^{-1}$, where the sign relates to the sense of loop shear, corresponding to a forward sigmoidal shape for positive and backward for negative $\alpha$.

We finally plotted projections of some 3-D model magnetic field lines onto the EIT images. One such example is shown in Fig. 9. The radiance images appear diffuse and hardly provide a clue about the coronal magnetic field. However, being guided by merely a few fieldlines one can much better imagine the field topology, and thus discern the grossly bipolar structure of the active region, which consists of two major bright coronal flux tubes bridging the two domains of opposite polarity. Before we go into further details of the observations, the extrapolation scheme and mathematical tools will be described.

\subsection{Computation of the 3-D coronal magnetic field}

\subsubsection{The fitting routine}

Direct observations of the magnetic field in the upper solar atmosphere are only available for singular cases, e.g. in the upper chromosphere for a newly developed active region observed by Solanki et al. (2003) and Lagg et al. (2004). To obtain the coronal magnetic field we have to extrapolate the routinely observed photospheric magnetic field into the corona. Potential fields (e.g. Schmidt 1964; Semel 1967) are completely determined by fixing the line-of-sight component of the photospheric magnetic field, but by definition necessarily do not provide good fits to the observed coronal emission structures. In principle, it is desirable to reconstruct the coronal magnetic field of active regions with the help of a non-linear force-free extrapolation, (e.g. Sakurai 1981; Roumeliotis 1996; Amari et al. 1997, 1999; Wheatland et al. 2000; Wiegelmann \& Neukirch 2003; Wiegelmann 2004). However, this requires vector magnetogram data as input at the photosphere. Unfortunately, the corresponding data are not available for the EIT and SUMER data discussed in this paper. In the case of linear force-free fields, the normal (e.g. Chiu \& Hilton 1977) or line-of-sight component (Semel 1988) is not sufficient to determine the field uniquely, and one therefore has the freedom to choose a value for the linear force-free parameter $\alpha$.

Our aim here is to compute the value of $\alpha$ by fitting 2-D projections of the magnetic field lines (with different values of $\alpha$ ) on the emitting plasma structures as observed by EIT. It is
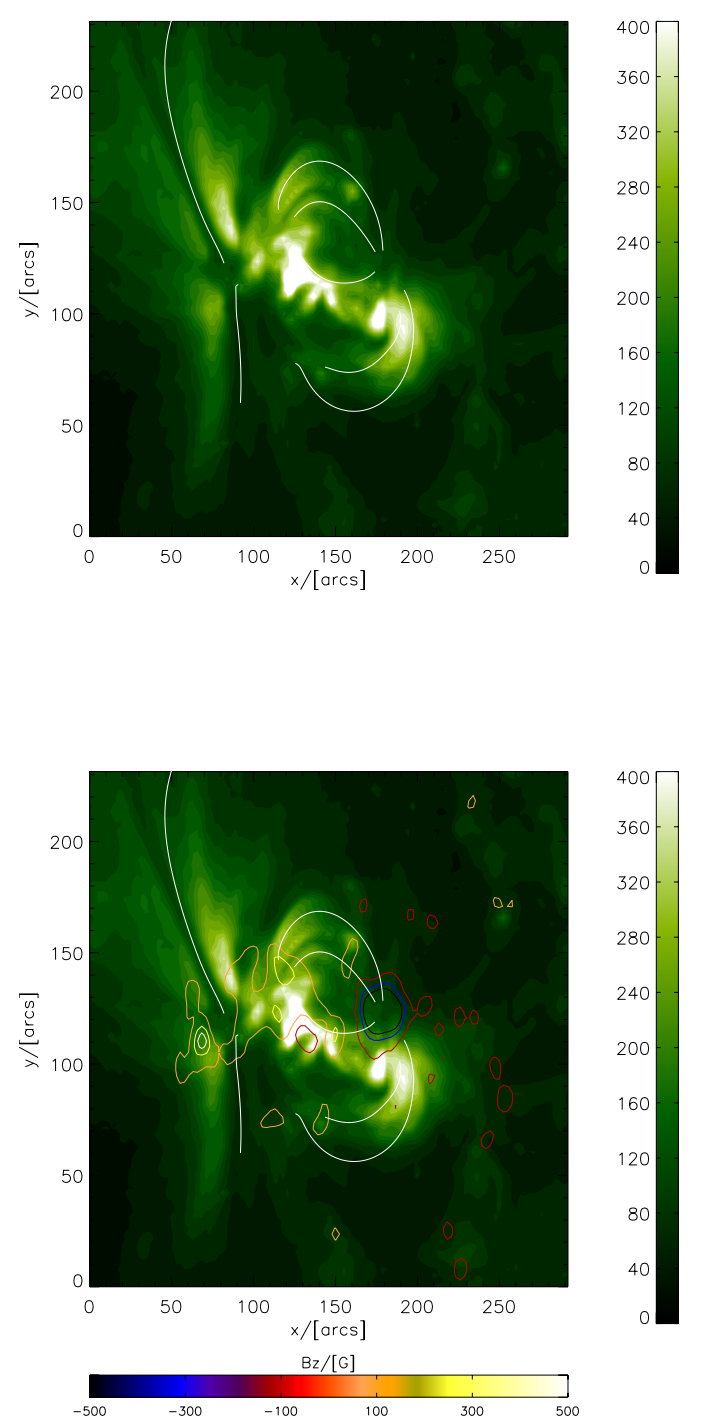

Fig. 9. Top: EIT picture of the active region AR 7953 and the 2-D projection of some reconstructed magnetic field lines, with the forcefree parameter times the scale length obeying: $\alpha L=-2.0, \quad(\alpha=$ $\left.-1.1 \times 10^{-8} \mathrm{~m}^{-1}\right)$. Bottom: the same picture, but also showing additional contours of the source magnetic field strength (in Gauss) on the photospheric boundary layer.

assumed that, due to the high conductivity and strong magnetization, the coronal plasma follows and thus outlines the field lines. This alignment of the magnetic field with the coronal plasma (the gradient of the plasma density is much higher perpendicular than parallel to the magnetic field) has been used by Wiegelmann \& Neukirch (2002) to compute the optimal value of $\alpha$ with respect to 3-D plasma loops computed with the help of dynamic stereoscopy developed by Aschwanden et al. (1999). The method was further developed by Carcedo et al. (2003) and used with 2-D projections of coronal loops observed by Yohkoh. The latter method requires that the foot point areas of the coronal loops are identified. This is not always possible in the EIT and SUMER images used here. Therefore, we further develop the optimization method here so that it also works well with loop segments, when parts of the loop, e.g. one or even both foot points, have not been identified. 
To do so we identified several loops (or parts of loops) by means of the emission patterns in the EIT images. The corresponding structures are two-dimensional with coordinates $(x, y)$, since no information regarding the height $z$ of the loops is available. We calculate the magnetic field lines by means of a fourth order Runge-Kutta field-line tracer. The field line integration starts at one point, say $\left(x_{0}, y_{0}\right)$, of the 2-D plasma loop. We then integrate in the $+\boldsymbol{B}$ and $-\boldsymbol{B}$ directions until the field line reaches the photosphere. The start height $z_{0}$ and the value of $\alpha$ for the field line integration are unknown a priori. We can vary these two parameters and thus find the optimal values. As a measure of the quality of the reconstruction, we compute the area between the 2-D projection of the magnetic field lines and the plasma loop. We find the optimal values of $\alpha$ and $z_{0}$ by minimizing this area. While the loop height $z_{0}$ is individual for each loop, the value of $\alpha$ has to be unique for all loops in order to be consistent with the linear force-free approach. In practice, we first calculated individual values of $\alpha$ for each loop and afterwards computed the optimal global value of $\alpha$.

The five closed loops shown in Fig. 9 have been computed with $\alpha \cdot L=-2.0$ and seem to outline the EIT loops quite well. We did not apply any fitting procedure to the open field lines. But the two resulting (calculated as well with the value $\alpha \cdot L=-2.0$ ) open field lines shown in Fig. 9 seem to fit the underlying EIT image well too. As we have identified $\alpha \cdot L=-2.0$ as the optimal global value of $\alpha$ within the linear force-free approach, we can now compute the magnetic field everywhere in the volume above the photospheric plane for which we have the magnetogram. Figure 10 shows the final result of this extrapolation with a suitable selection of closed and open magnetic field lines, chosen to fill space in three dimensions.

\subsubsection{Seehafer solution for linear force-free field}

We use the method of Seehafer (1978) for calculating the linear force-free field for a given magnetogram and a given value of $\alpha$. This method gives the components of the magnetic field in terms of a Fourier series. The measured magnetogram, which covers a rectangular region extending from 0 to $L_{x}$ in $x$ and 0 to $L_{y}$ in $y$, is then artificially extended to a rectangular region covering the square from $-L_{x}$ to $L_{x}$ and $-L_{y}$ to $L_{y}$. This supplementation is achieved by taking an antisymmetric mirror image of the original magnetogram in the extended region, i.e. we assume that $B_{z}(-x, y)=-B_{z}(x, y)$ and $B_{z}(x,-y)=-B_{z}(x, y)$. The advantage of taking an antisymmetric extension of the original magnetogram is that the extended one is thus automatically flux balanced. A Fast Fourier Transformation (FFT) scheme is used to determine the coefficients of the series. The components of the magnetic field can thus mathematically be expressed as:

$$
\begin{aligned}
B_{x}= & \sum_{m, n=1}^{\infty} \frac{C_{m n}}{\lambda_{m n}} \exp \left(-r_{m n} z\right) \\
& \times\left[\alpha \frac{\pi n}{L_{y}} \sin \left(\frac{\pi m x}{L_{x}}\right) \cos \left(\frac{\pi n y}{L_{y}}\right)\right. \\
& \left.-r_{m n} \frac{\pi m}{L_{x}} \cos \left(\frac{\pi m x}{L_{x}}\right) \sin \left(\frac{\pi n y}{L_{y}}\right)\right]
\end{aligned}
$$

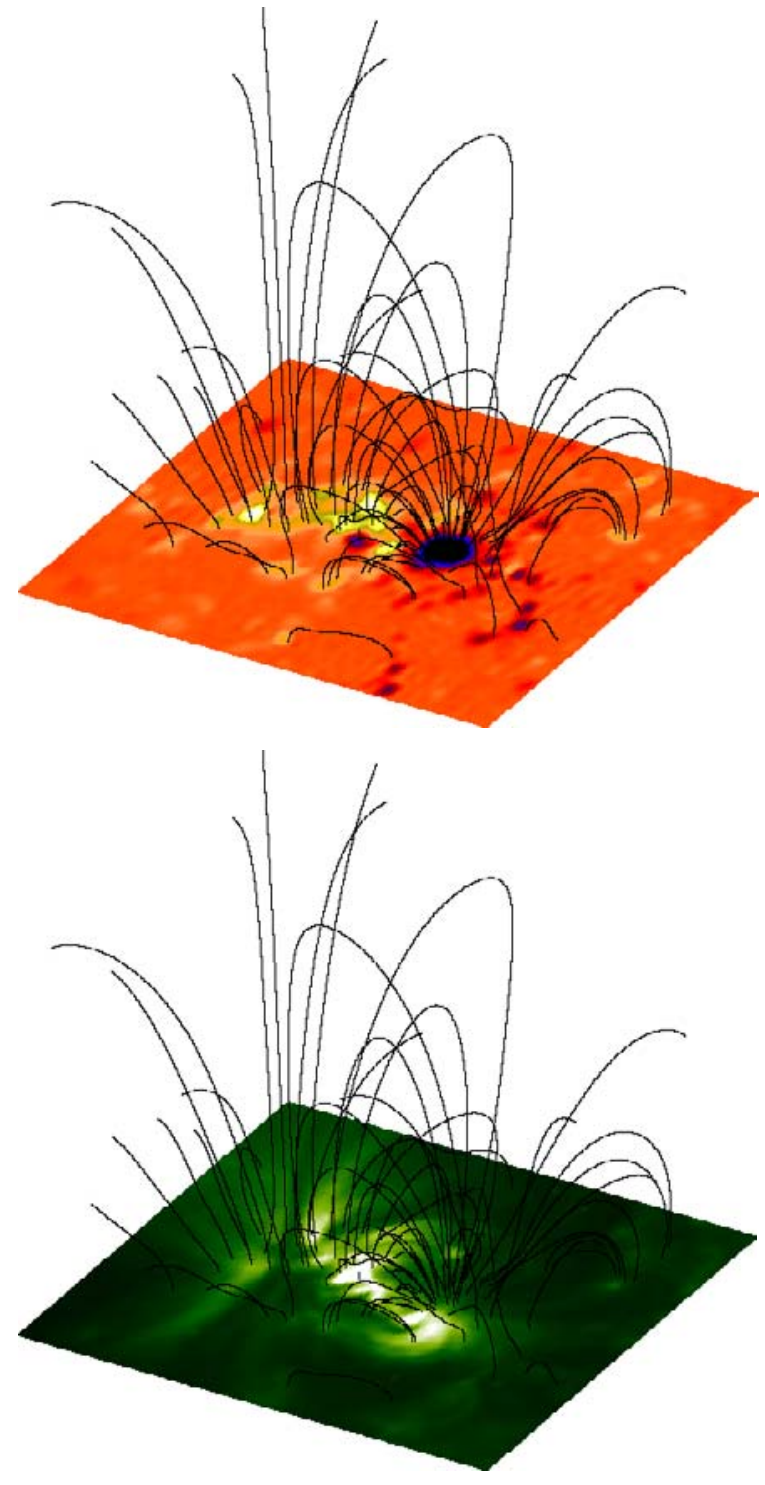

Fig. 10. The magnetic field topology in three dimensions. Magnetic field lines with $\left|B_{z}\right|>50 \mathrm{G}$ on the photosphere are plotted. Here the colour coding, respectively, represents the magnetic field strength in the top panel and the intensity of the EUV emission in the EIT image projected onto the bottom of the coronal box in the bottom panel.

$$
\begin{aligned}
B_{y}= & -\sum_{m, n=1}^{\infty} \frac{C_{m n}}{\lambda_{m n}} \exp \left(-r_{m n} z\right) \\
& \times\left[\alpha \frac{\pi m}{L_{x}} \cos \left(\frac{\pi m x}{L_{x}}\right) \sin \left(\frac{\pi n y}{L_{y}}\right)\right. \\
& \left.+r_{m n} \frac{\pi n}{L_{y}} \sin \left(\frac{\pi m x}{L_{x}}\right) \cos \left(\frac{\pi n y}{L_{y}}\right)\right] \\
B_{z}= & \sum_{m, n=1}^{\infty} C_{m n} \exp \left(-r_{m n} z\right) \cdot \sin \left(\frac{\pi m x}{L_{x}}\right) \sin \left(\frac{\pi n y}{L_{y}}\right)
\end{aligned}
$$

with $\lambda_{m n}=\pi^{2}\left(m^{2} / L_{x}^{2}+n^{2} / L_{y}^{2}\right)$ and $r_{m n}=\sqrt{\lambda_{m n}-\alpha^{2}}$. The expansion coefficients $C_{m n}$ are obtained by comparing Eq. (3) for $z=0$ with a FFT of the magnetogram data. The numerical method has to cut off the Fourier series at some maximum 
values, $m_{\max }$ and $n_{\max }$. To normalize $\alpha$ we choose the harmonic mean $L$ of $L_{x}$ and $L_{y}$, which is defined by $2 / L^{2}=\left(1 / L_{x}^{2}+1 / L_{y}^{2}\right)$.

\section{A comparison between magnetograms and Dopplermaps of AR 7953}

\subsection{Comparison between magnetogram and Dopplergram}

We return to the AR 7953 and now discuss the details of its magnetogram in relation with the Dopplergram. The EIT image of this AR was shown before in Fig. 9, and the associated magnetic field obtained by extrapolation was already illustrated in Fig. 10, by means of showing two bundles of closed and open field lines. A corresponding SUMER Dopplergram in the Ne VIII $(\lambda 77 \mathrm{~nm})$ line together with some selected field lines is now given in Fig. 11. The bottom frame of this figure shows additional contours of the magnetic field strength on the photosphere. Only a few field lines where chosen such as to clearly signify the connections between the Doppler shifts and the field topology.

Inspection of the bottom frame of Fig. 11 shows that the region of the bipolar AR with negative field polarity (blue contours) is rather concentrated in an area corresponding to the visible sunspot, and correspondingly the magnetic field lines form a compact bundle there, whereas the region of positive polarity is wide spread and consist of a group of spotty fields (yellow contours on the left), which are at the origin of open as well as closed field lines. There are two dark red and blue Dopplershift domains in the location of the sunspot proper, separated by rather sharp boundaries between these regions of up and down flows. This feature is similar to what we already found in the two ARs shown without their fields in Figs. 5 and 7, where distinct limits between red and blue domains were found. The constriction of the field line bundle coincides with significant neon ion outflow (blue shift) in an area which has the largest negative $B_{z}$.

Note that the two flux tubes connecting the two magnetic poles of this bipolar AR have mostly a Doppler red shift on one and blue shift on the other leg, indicating an ordered flow between both feet as expected in a steady siphon flow. Inspection of the few open (with respect to the extrapolation box) field lines on the left of the figure reveal thin adjacent flux tubes with opposite up and down flows coexisting near to each other. Apparently, material may rain down on some field lines and stream up on the neighbouring ones (as seen here in projection) being just a few arcseconds apart. At some locations these gradients occur at a current sheet, such as at $y=120^{\prime \prime}$ and $x=130^{\prime \prime}$, where adjacent reddish and orange contours indicate a clear magnetic field polarity reversal. There are also a few isolated spots of localized blue shifts (for example near the site with coordinates $x=250^{\prime \prime}$ and $y=90^{\prime \prime}$ ), which coincide with strong inward fields there. In conclusion, the AR field is highly structured and associated with a complex pattern of Dopplershift or mass motion, similar to what we concluded for the other two ARs before by only inspecting Doppler maps.

To get a better overall impression of the magnetic field topology we show in Fig. 12 the bipolar AR again, with many
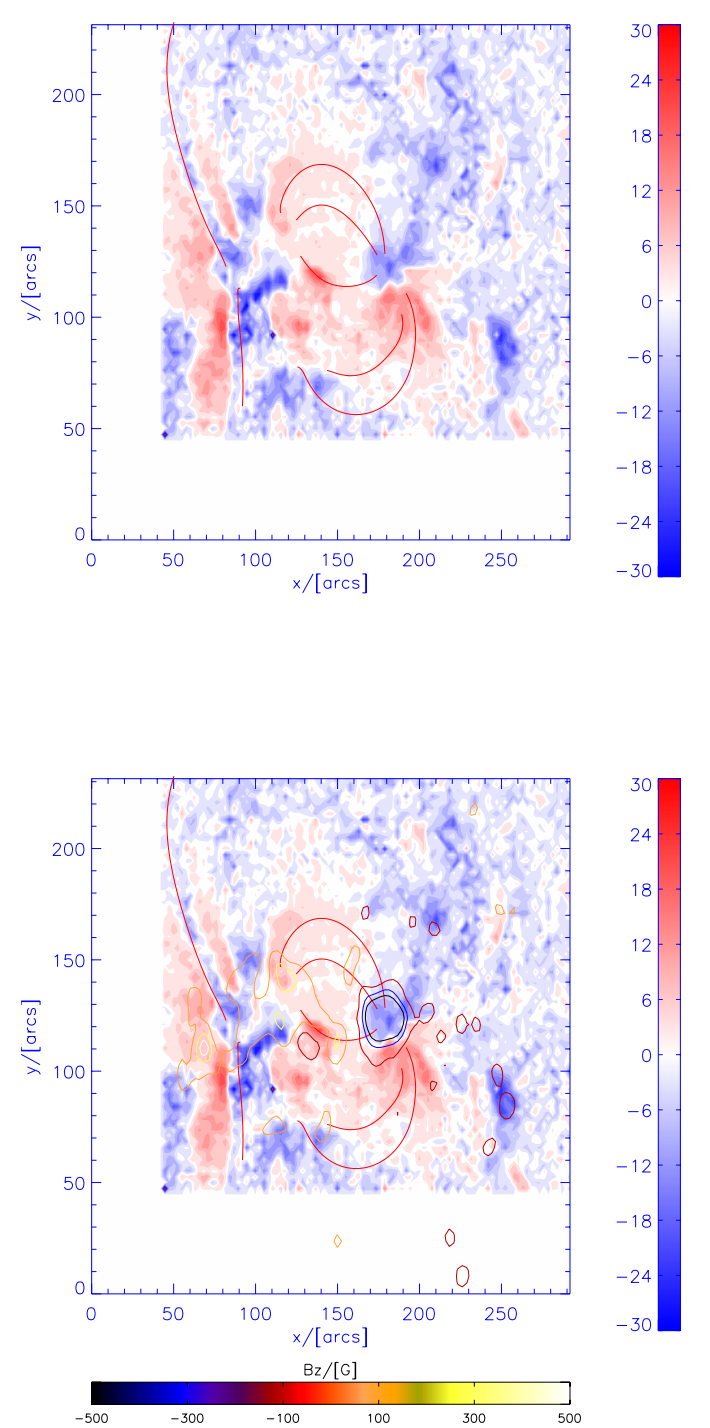

Fig. 11. SUMER Dopplergram in Ne VIII $(\lambda 77 \mathrm{~nm})$ and a 2-D-projection of some reconstructed magnetic field lines with $\alpha \cdot L=-2.0,\left(\alpha=-1.1 \times 10^{-8} \mathrm{~m}^{-1}\right)$. The bottom picture shows additional contours of the magnetic field strength on the photosphere. For the white stripes in the left and lower part of the picture SUMER data are not available.

projected field lines and Dopplershift contours overlaid. In the top frame the magnetic fields are plotted for magnitudes larger than $200 \mathrm{G}$, and in the bottom frame the magnetic fields with magnitude smaller than $200 \mathrm{G}$. The two figures complement each other so that a complete impression of the magnetic connectivity is obtained by their superposition. Note the close correlation between the Dopplershift pattern and the field, being either closed or open. The stronger fields connect the two poles of the bipolar AR and close among the poles, with the highest magnetic flux concentration in the right sunspot showing the most distinct blue shift in the neon line like in Fig. 11. The weaker fields reach out into the spot's neighbourhood and originate from regions having both red or blue shifts alike.

Apparently, the simple bipolar nature of this AR on large scales is clearly revealed in Fig. 12. However, detailed inspection of the previous figures shows that there are many 

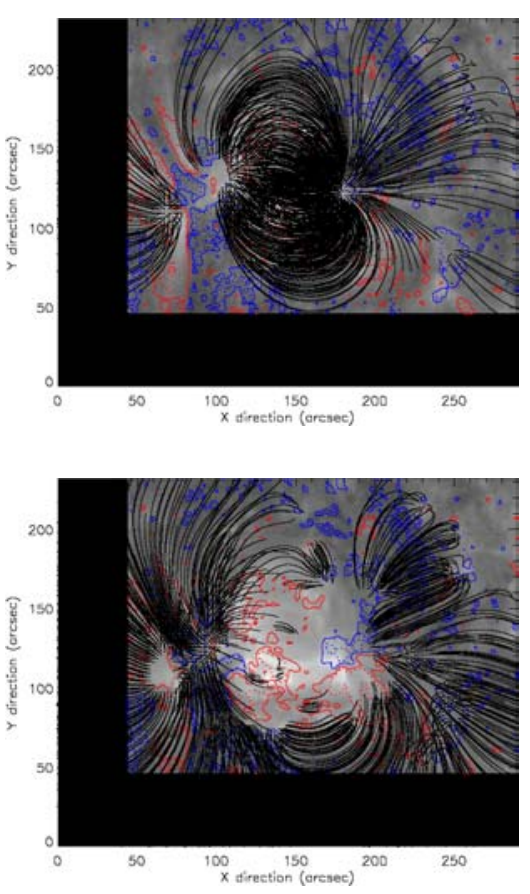

Fig. 12. Illustration of the topology of magnetic field lines in the AR. Top: magnetic fields with magnitude larger than $200 \mathrm{G}$; Bottom: magnetic fields with magnitude smaller than $200 \mathrm{G}$. The background images show the intensity map of the Ne VIII line, with its Dopplershift contours overlaid. Note the correlation between the Dopplershift pattern and the field being either closed or open.

smaller-scale parts of this AR which have a close correspondence between their fields and motions (shifts) down to the arcsecond scale.

The patches of red shift in the middle of the bottom frame of Fig. 12, since they are shown in projection, correspond to the upper and apex parts of the system of closed loops shown in the top frame, thus indicating coronal material flowing down the loops mainly to the left-hand magnetic flux concentration. In contrast, the very blue patches in the left corner of the top frame are closely associated with a locally open field, as is visible in the left corner of the bottom frame. They also correspond to a low intensity in the Ne VIII line. The same close relationship between Doppler-blueshift and low intensity was typically found in coronal holes, where the magnetic field is globally open (Hassler et al. 1999; Wilhelm et al. 2000; Xia et al. 2003).

ARs were recently identifed as sources of the solar wind by Liewer et al. (2003). They were able, by extrapolation to the solar magnetic source surface, to correlate in-situ solar wind measurements with the ARs evident in magnetograms. Furthermore, they inferred the dark lanes or holes adjacent to the ARs in EUV images as the possible plasma sources related with open field lines. Thus they could trace one possible origin of the slow solar wind. Here we corroborate their findings, identifying coronal outflow in ARs directly by means of Doppler blue-shifts, like it was done for $\mathrm{CHs}$ before in the three above-cited references.

\subsection{Comparison between field geometry and Dopplergrams at various heights}

As the previous figures clearly showed, the magnetic field of the AR is highly structured on all scales, down to the resolution of the imagers and spectrometers of $1^{\prime \prime}$ to $2^{\prime \prime}$, and so is the plasma flow pattern as it appears in various shifts of ion emission lines, in particular in the one of neon forming nearly at coronal temperature. As is expected, with height in the corona the structure of the field simplifies and the plasma structures while heating up become more extended and diffuse in the coronal images. These changes take place within a few $\mathrm{Mm}$ in altitude, a height range over which also the fine structures still seen on the disk become more blurred or disappear, whereby the field becomes more uniform.

Figure 13 presents an overview of the evolution with height of the magnetic field $B_{z}$ component (shown on the left sides) and the field angle defined by $\tan ^{-1}\left(\sqrt{B_{x}^{2}+B_{y}^{2}} /\left|B_{z}\right|\right)$ (shown on the right sides) at the heights: $0,3,5$, and $10 \mathrm{Mm}$. In the left panels of this figure, the very dark regions correspond to the sunspot umbra dominated by negative polarity of magnetic field. One can infer from close inspection of these images that the flux tube expands with increasing height. In the central region of the sunspot, strong blueshifts of the Ne VIII line dominate, while a velocity shear boundary can be identified at its southern edge. On the left side of the sunspot, magnetic fields with positive polarity pertain, in which several locations are apparent with strong velocity shear seen in the Ne VIII line. From the right panels of the figure one can conclude that the strong velocity shears are mainly associated with the boundaries between the vertical field-line bundles and oblique ones (inclined at a large angle).

Clearly, both maps of the magnetic field strength and of the angle in Fig. 13 indicate that the magnetic field topology becomes more uniform with increasing height. Small-scale magnetic field fluctuations on the photosphere cancel out at low heights by forming small-scale closed loops, and only the large-scale structures of the magnetic field survive at greater heights. This behaviour is comparable with that of a multipole representation, where at large distance from the source only the lowest-order multipole is significant.

Further close inspection of the right panels of Fig. 13 shows that the regions of strong neon blueshifts are mostly associated with the larger patches of vertical field orientation, such as in the sunspot itself and in the broader counterpart to its left, both together making up the bipolar AR, as well as in the dark patch in the right bottom corner. In contrast, the grey and white regions correspond to more horizontal fields and are closely related with neon red shifts, indicating perhaps mostly downflows in canopy-type loop segments.

Note in the upper frames of Fig. 13 that the C IV line somehow shows a more mixed Dopplershift pattern. Yet, the related velocity shears are not as sharp as those of neon. Strong carbon blueshifts are also found in the central region of the spot, while in many other regions with vertical field lines we find redshifts. The different behaviour of these two lines may indicate that the 

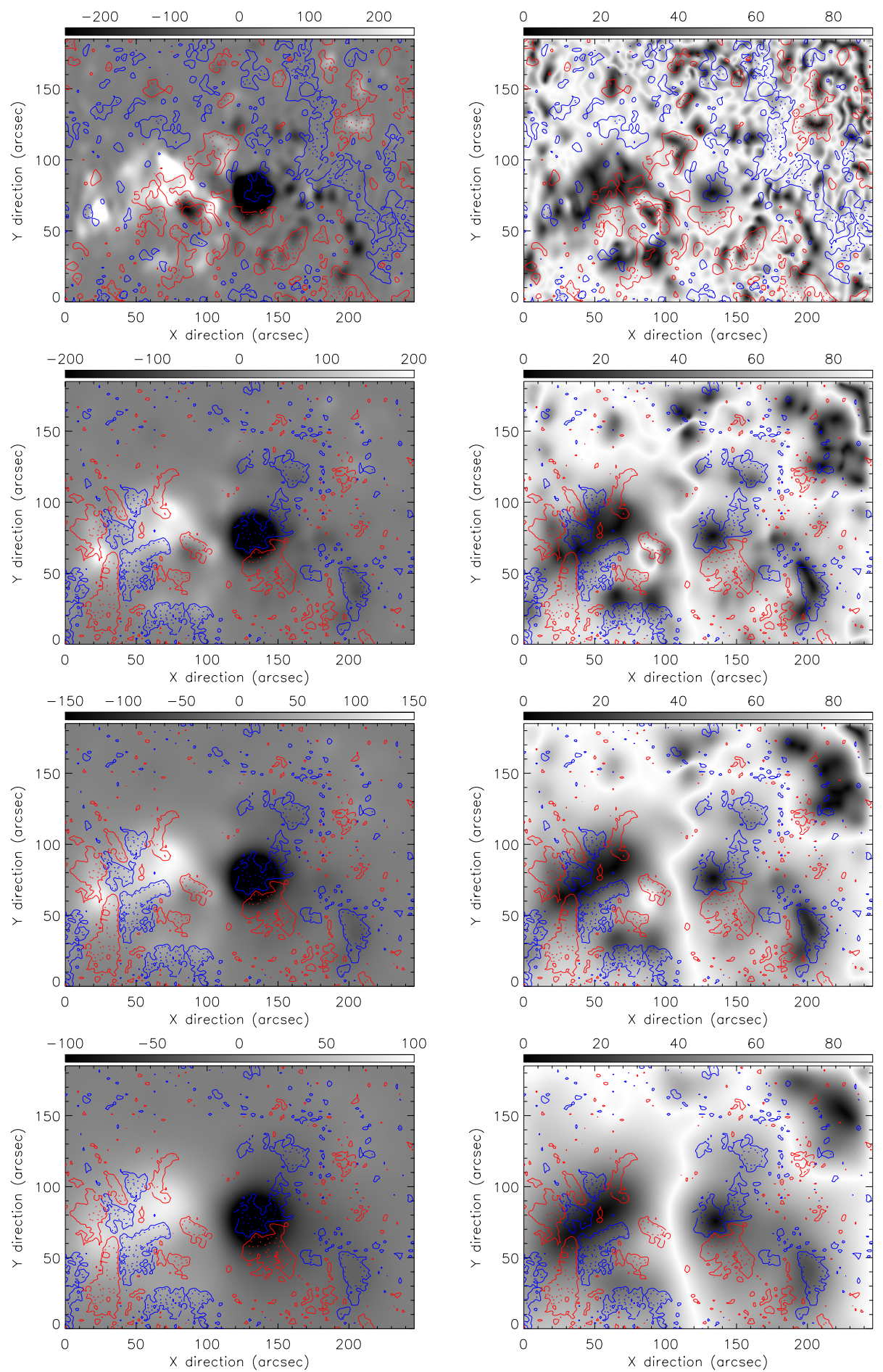

Fig. 13. Comparisons of magnetic field strength maps, angle maps and Dopplermaps of the active region at four different heights in the atmosphere. Heights from the top to the bottom: 0,3,5,10 Mm. The coloured contours correspond to shifts of $\pm 10 \mathrm{~km} \mathrm{~s}^{-1}$ (dotted lines) and $\pm 5 \mathrm{~km} \mathrm{~s}^{-1}$ (continuous lines). Left: magnetograms with Dopplershift contours of the C IV (only upper frame) and Ne VIII (all other frames) lines overlaid. Right: field angle maps with the same contours overlaid.

carbon emission mostly comes from plasma confined in lowlying, small-scale loops.

In the upper right corners of the top right frames of Fig. 13 one finds meso-scale (of about $10^{\prime \prime}$ in size) weak-field magnetic structures with varying field inclinations. This region is restructuring with height and becomes more uniform at $10 \mathrm{Mm}$ (bottom panel), where the small structures seem to have merged and form a coherent large patch indicating more vertical field lines. This is the footpoint domain of the many already mentioned arches that all originate in the sunspot proper. The carbon emission in these small structures is mostly red shifted (as shown below in Fig. 15) and indicates lower-temperature down-flowing plasma confined in small loops that populate the footpoint regions of the larger loops. 
The previous discussion has amply demonstrated how important it is to have both magnetograms and Dopplergrams when analysing and interpreting coronal images in various lines in projection against the solar disk. In Figs. 6 and 7 we have illustrated the complex radiance and Doppler shift patterns (in carbon and neon) for AR 8534, without having magnetic fields available. The AR 7981 appears similarly complex and reveals very sharp boundaries between distinct domains of blue and red shifts at various heights (corresponding to lines at different formation temperatures). For the present case of AR 7953 we have the extrapolated magnetic field and thus can compare the Dopplershift gradients with the underlying magnetic field topology.

Let us finally return to Fig. 13 to scrutinize the Dopplershift pattern in the positive pole (left white spot) of the bipolar AR. It has a uniform black counterpart in the right frames, indicating a mostly vertical orientation of the field. It can clearly be seen that this pole is the origin of adjacent up and down flows. They are located on neighbouring field lines of the same polarity and just a few arcseconds apart. This indicates that a highly structured flow pattern is associated with this pole. Close inspection of Figs. 11-13 together, shows that there is a slight tendency of red shifts to occur in stronger closed field lines and blue shifts on weaker open field lines, which according to Fig. 10 reach greater heights in the corona.

\section{Coronal mass flux and current density}

\subsection{Geometry of the magnetic flux tube}

The magnetic field modelling procedure described in a previous section allows one to calculate magnetic flux tubes and their geometry at any location in the numerical extrapolation box above the photosphere. The area function or cross section of a flow or flux tube is the key parameter to estimate and quantify the coronal mass flux, and therewith to estimate the local and global circulation of matter in the corona. In the outer panels of Fig. 14 we show one example of a flux tube as constructed from the force-free model. It is illustrated either by a set of rings, indicating its varying cross sections with height (top frame), or as a sausage-type structure, emphasizing its longitudinal extension (bottom frame). For a further illustration of the flux tube geometry, especially its diameter and cross-section function, see the middle frames of Fig. 14, which show the expansion of the flux tube with height and its constriction at the foot points, as well as its normalized transverse extent versus coordinate along the loop.

The geometry thus inferred for a typical flux tube in an observed AR, through the property that the field is divergence free, defines the tube area function which in turn constrains the mass flow in the expanding flux tube. (The field is characterized by $\nabla \cdot \boldsymbol{B}=0$, and consequently $B A$ is constant along a magnetic flux tube of cross-section $A$.) The loop expansion factor in the third frame of Fig. 14 is consistent with values for a force-free sheared arcade, according to the thickness variations of coronal loops composed in Fig. 5.14 in the book of Aschwanden (2004) and references therein. Force-free twisted flux tubes were observed to reveal hardly any expansion, and were calculated to
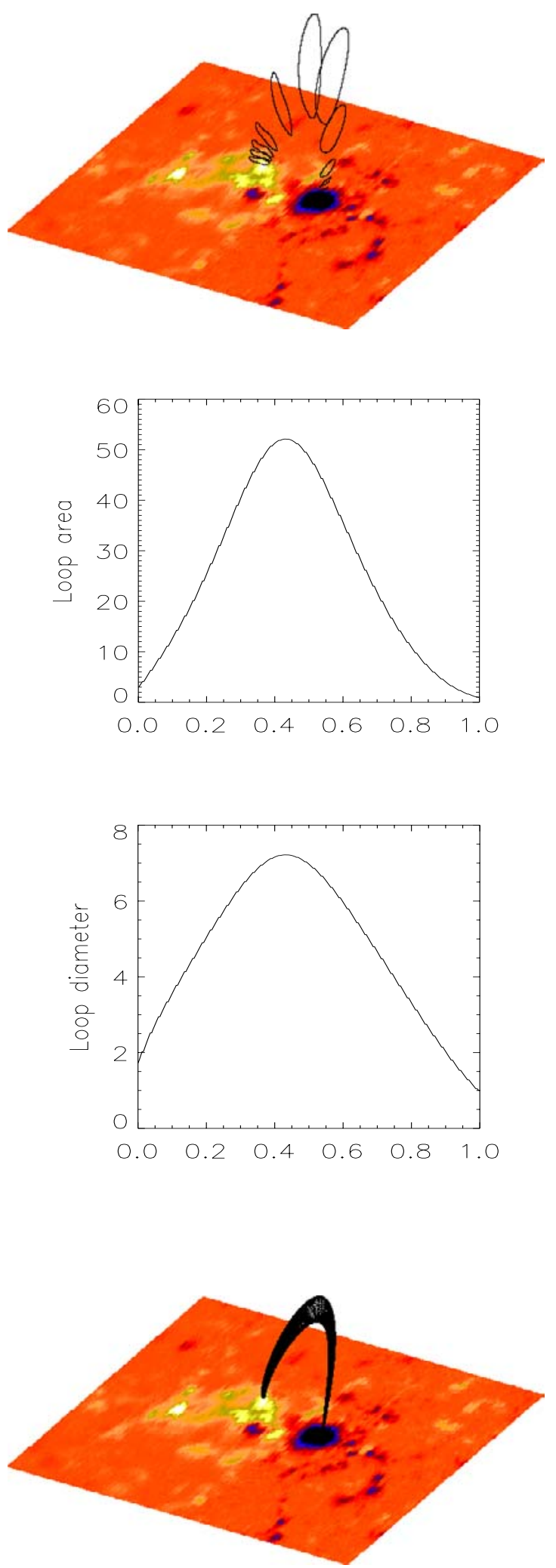

Fig. 14. Illustration of a magnetic flux tube associated with the exemplary active region AR 7953 shown previously. Top: individual perpendicular cuts through the tube, indicating the increasing size of the area with height in the atmosphere. Second row: loop area in arbitrary units (unit area at the right footpoint). Third row: linear loop diameter in arbitrary units (unity at the right footpoint): Bottom: visualization of the entire longitudinal extent of the flux tube, showing its expansion in diameter with height and constriction at the foot points. 


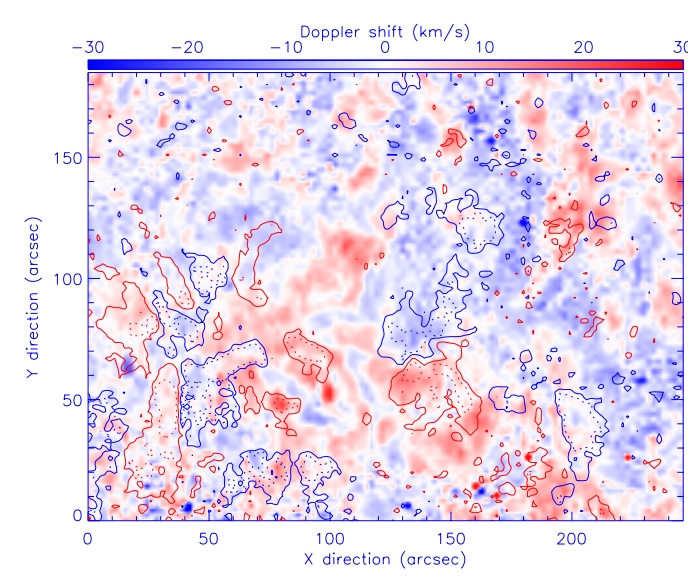

Fig. 15. Dopplergram of the C IV line overlaid by Dopplershift contours of the Ne VIII line, illustrating the difference in plasma velocities as seen, respectively, in the lower and upper transition region. The colour bar gives the coding for the carbon shift. The neon contours correspond to shifts of $\pm 10 \mathrm{~km} \mathrm{~s}^{-1}$ (dotted lines) and $\pm 5 \mathrm{~km} \mathrm{~s}^{-1}$ (continuous lines). Note the close correlation between the carbon-shift background colour pattern and the neon-shift coloured contours.

have almost constant diameters due to the twist-induced constriction. Therefore, twist seemed to have a minor effect on the flux tube studied here.

\subsection{Estimates of the coronal mass flux}

If the Doppler shift seen in an EUV emission line of a minor ion can be considered as being caused by coronal bulk mass motions (i.e. motions of protons and alpha particles), which is tacitly assumed to be the case in most Doppler shift studies, then it can be directly used to mark the coronal plasma flow. The constriction of the lower segments of our model flux tube shown in Fig. 14 has the consequence that in the chromosphere and lower transition region, due to the higher plasma density, the velocity of a steady flow must be lower there than in the upper transition region. (A steady plasma flow is characterized by $\nabla \cdot(\rho \boldsymbol{v})=0$, and consequently $\rho v A$ is constant along a flux tube of cross-section $A$.) Such a trend can be inferred from Fig. 15 in which a Dopplergram of the C IV line is overlaid by the Dopplershift contour of the Ne VIII line. This plot illustrates the different plasma velocities as seen, respectively, in the lower and upper transition region.

Let us return for a while to the Dopplergrams studied in connection with the height variation of the model magnetic field as demonstrated in Fig. 13. We show a smaller segment of the same area of the AR including the sunspot in Fig. 15, which is a combined Dopplergram of the shifts of the Ne VIII and C IV line. Recall that the sunspot center is located at coordinates $y=80^{\prime \prime}$ and $x=130^{\prime \prime}$. It is clearly discernable by the small closed contours of a strong neon blue shift, surrounded to the left by three large patches of neon red shifts.

In Fig. 15 one can see that the Doppler shift patterns of the C IV line generally follow those of the Ne VIII line. Please bear in mind that the Dopplergrams shown here are calculated relative to the average shifts, which in the absence of absolute

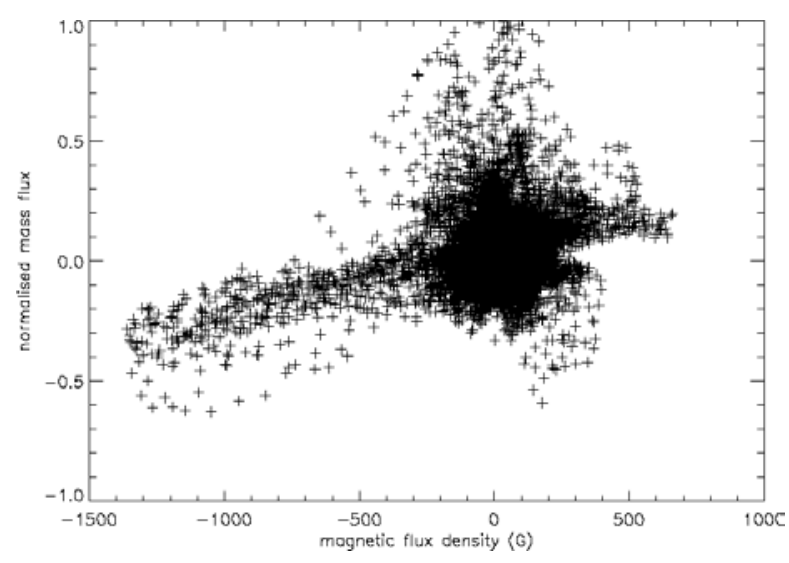

Fig. 16. Scatter plots of the mass flux density derived from the Ne VIII line shift used as a marker of plasma flow, plotted as a function of magnetic flux density derived from the data obtained by NSO/KP. Note that the unit of the mass flux is set at arbitrary values, with negative values indicating upflows and positive downflows.

wavelength calibration for this data set were evaluated over the whole FOV of SUMER. This procedure is reasonable for the Ne VIII line, because previous studies showed that its average shift is near zero in QS regions, while the C IV line has a clear red shift between 5-10 $\mathrm{km} \mathrm{s}^{-1}$ (see e.g. Teriaca et al. 1999; Xia 2003).

If taking this average red shift into account, we find that the so calibrated C IV line then has a very small blue shift (less than $5 \mathrm{~km} \mathrm{~s}^{-1}$ ) in the sunspot center, where the Ne VIII line shows large blue shifts (larger than $10 \mathrm{~km} \mathrm{~s}^{-1}$ ). Small blue shifts of this line were previously found in sunspot umbrae with the Ultraviolet Spectrometer and Polarimeter (UVSP) on the Solar Maximum Mission (SMM) (Gurman \& Athay 1983), and significant values from $15-40 \mathrm{~km} \mathrm{~s}^{-1}$ were found by Winebarger et al. (2002) in the sunspot of the AR 8534, of which we showed Doppler maps in Fig. 7.

We will now derive estimates of the coronal mass flux density. Following the procedure applied before by Xia et al. (2003), we use the quantity $v \sqrt{I}$, which is the square-root of the line intensity ( $I \sim n_{\mathrm{e}}^{2}$ used as a proxy for the squared plasma density) multiplied by the Dopplershift, $v$, to obtain a proxy for the plasma mass flux. This approximate quantity is here simply referred to as mass flux and plotted in Fig. 16, which gives this mass flux versus the magnetic field flux density as obtained from the NSO/KP data. The unit of the mass flux is set arbitrarily. For coronal holes typical (unsigned) values were estimated by Xia \& Marsch (2003). They found a proton flux of $(2.5-6.3) \times 10^{17} \mathrm{~m}^{-2} \mathrm{~s}^{-1}$ at photospheric field strengths of (1-3) G. For the (by an order of magnitude higher) typical density of $10^{15} \mathrm{~m}^{-3}$ in ARs we obtain, with the Doppler speed ranging between (10-30) $\mathrm{km} \mathrm{s}^{-1}$, a coronal proton flux of $(1-3) \times 10^{19} \mathrm{~m}^{-2} \mathrm{~s}^{-1}$, which is substantially larger than in the polar or equatorial coronal holes, from which the fast solar wind originates, but consistent with the magnetic field also being larger by about two orders of magnitude.

Inspection of Fig. 16 shows, for magnetic fields with a magnitude larger than $200 \mathrm{G}$, a clear positive correlation between 


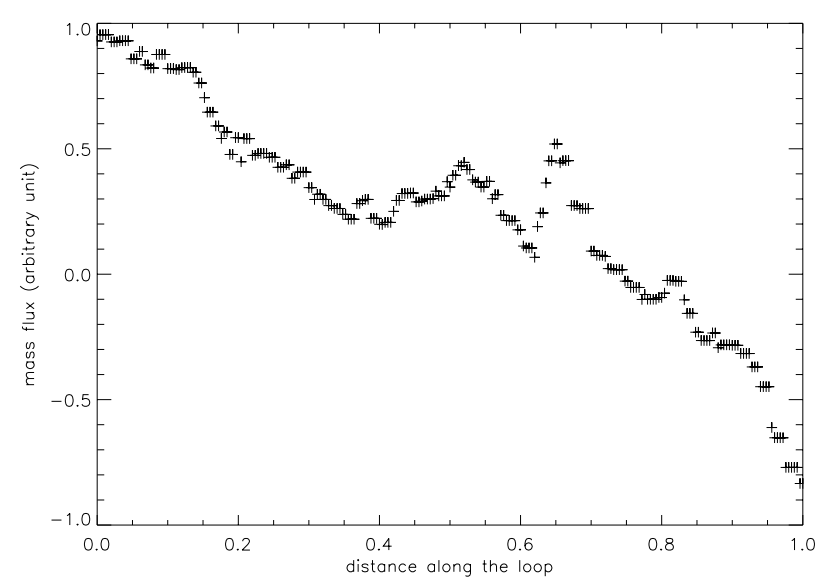

Fig. 17. Mass flux densities in arbitrary units as inferred in a coronal loop as a function of normalized coordinate along the loop. For the loop geometry see the previous figures. The averaged data almost form a straight line.

mass flux and magnetic field. Because the conspicuous loops have photospheric magnetic fields with magnitude larger than $200 \mathrm{G}$ (see Fig. 17), our findings may indicate that the mass flux in a large loop is directly associated with the magnetic flux. As expected, the outward mass flows correspond to a negative polarity of the field, originating mainly from the sunspot, while the inward mass flows are associated with a positive polarity of the field, corresponding to the opposite legs of the large loops connecting to the spot. Apparently, there is a significant almost linear correlation between the mass flux and magnetic flux, with a large statistically uncorrelated cloud of data points superposed on this trend around the origin of the scales in the weak field domain.

Recent TRACE observations gave evidence that EUV coronal loops at $T \approx 1-1.5 \mathrm{M} \mathrm{K}$ in active regions had enhanced densities, enhanced pressure scale heights and flat (filter ratio) temperature profiles as compared with predictions from a static equilibrium theory (e.g. Rosner et al. 1978; Serio et al. 1981). Winebarger et al. (2001) found lower limits for the flow velocity between 5 and $20 \mathrm{~km} \mathrm{~s}^{-1}$. By comparison of the observed intensities with quasi-static models, the authors concluded that the observations can only be explained by a mass flow from the chromosphere into the corona. The nature of these flows remained unclear. Patsourakos et al. (2004) investigated, whether an inclusion of a stationary mass flow could explain the discrepancies between TRACE-observations and static loop models. The authors concluded that stationarity is not sufficient, but that more likely time-dependent heating processes are important.

The plot in Fig. 17 is done without any geometrical correction. We see large mass flux densities at the two legs of the loop and a much smaller value in the middle of the loop around the apex. Although we have tried, it is difficult to further improve this analysis quantitatively, e.g. by considering the detailed geometry of the loop, because of its 3-D geometry as discussed above, and also owing to possible contamination of the local emission in the Ne VIII line by neon emission coming from other structures along and adjacent to the loop.

The overall pattern of the flow may still be compatible with a stationary siphon flow, whereby mass enters the loop on one side and continuously flows to the other, implying that the flow direction has an opposite sign in the two legs, which is observed, but also a finite flow at the loop apex, which is not observed. However, in our case the inferred low mass flux in the middle of the loop is perhaps due to a line-of-sight effect (the velocity here is more horizontal so that we detect only a very small vertical component). For another explanation, we like to remind of a scenario invoking asymmetric ballistic flow under gravity, whereby the mass flows fast into the tube on one side, slows down towards the top (like in a fountain), and while some matter is leaking over the apex, is then accelerating upwards towards the opposite leg of the loop.

What can we say about the mass reflux to the chromosphere? It appears difficult to find direct evidence for that in chromospheric lines, because the electron density there is about hundred times the density in the corona. Therefore if the mass flux is conserved, the plasma velocity expected at chromospheric heights will be only several percent of that in the corona. Such a small value for the Dopplershift, of the order or below $1 \mathrm{~km} \mathrm{~s}^{-1}$, is practically impossible to measure with SUMER.

\subsection{Estimates of the coronal current density}

The field extrapolation described in the previous sections allows one to construct for any field line the height profile of the field strength $B$, and as shown above, to infer by using magnetic flux conservation the flux tube cross-section function. Moreover, given the alpha parameter is known, then the basic force-free relation (with the free space magnetic permeability $\left.\mu_{0}\right)$,

$\boldsymbol{j} /\left(\mathrm{A} \mathrm{m}^{-2}\right)=\frac{\alpha / \mathrm{m}^{-1}}{\mu_{0}} \boldsymbol{B} / \mathrm{T}=\frac{\alpha / \mathrm{m}^{-1}}{\mu_{0}} 10^{-4} \boldsymbol{B} / \mathrm{G}$,

enables the coronal current density to be inferred from the model field. This was for many active regions first done by Pevtsov et al. (1997). They obtained typical values in the range $j_{z}=(1-15) \mathrm{mA} \mathrm{m}^{-2}$ for a field $B_{z}$ of several hundred gauss. In Fig. 18 we present our results for the same flux tube discussed above. Apparently, current densities in the (0.1-1) $\mathrm{mA} \mathrm{m}^{-2}$ range are typical for the force-free, slightly-sheared fields in the present active region.

According to Eq. (4), the height profile for the magnetic field strength is apart from a constant the same as for the current. Note the asymmetry of the profiles shown in Fig. 18. Winebarger et al. (2002) argued that the steady flow detected in the AR 8534 (see our Figs. 6 and 7 again) would be expected for an asymmetric heating function that is proportional to the footpoint field strength. The same argument may apply the the AR loop studied here. However, detailed modelling would be required to verify this speculation. 

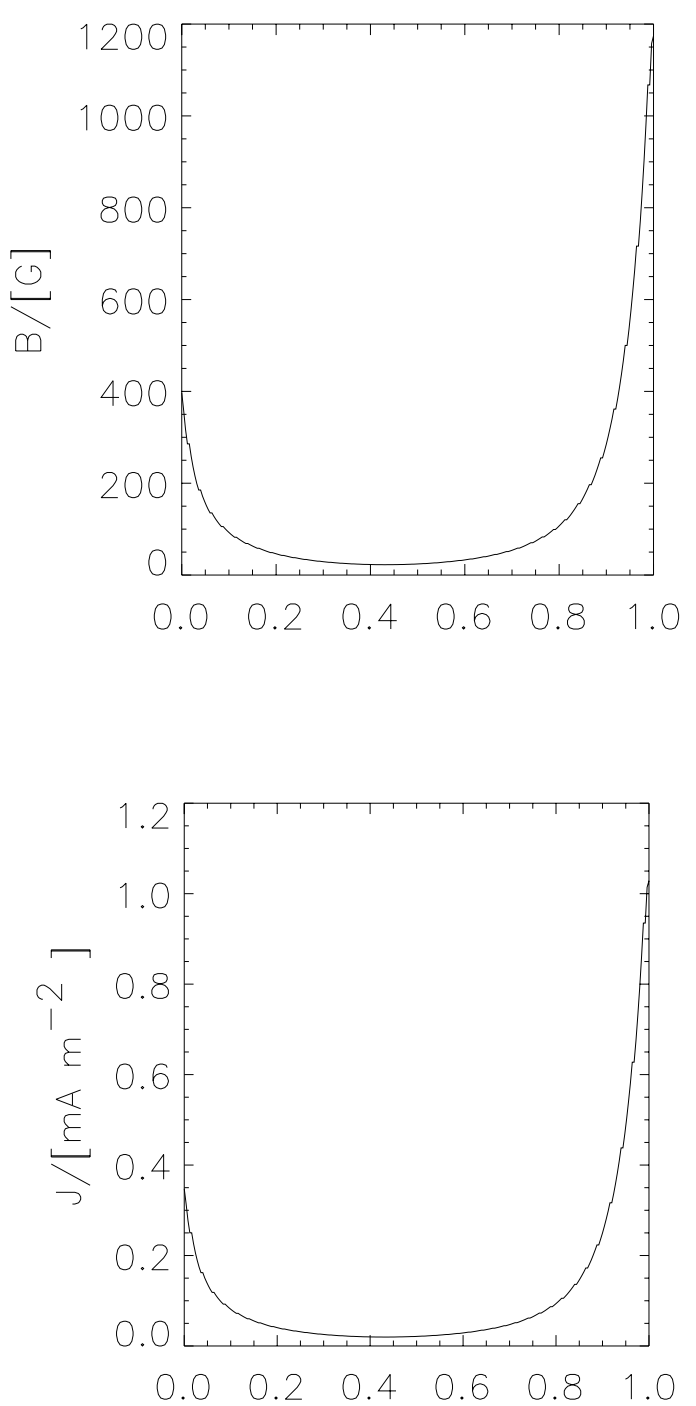

Fig. 18. Top: illustration of the magnetic field strength in dependence on the normalized coordinate along a single magnetic flux tube associated with the model magnetic field of the exemplary active region AR 7953 shown previously. Bottom: inferred current density distribution in the flux tube, showing a spatial asymmetry and the current concentration at one foot point.

\section{Summary and conclusion}

By combining observations made by SUMER aboard SOHO and photospheric magnetic field measurements made by MDI and at NSO/KP, the plasma flows and the magnetic field structures in several solar active regions were studied. In particular, we analysed the relationship between the ultraviolet emission line parameters, such as radiance and Doppler shift, and the topology of the background magnetic field, which was obtained from force-free field extrapolations of measurements made in the photosphere.

We may summarize our results as follows. We have studied three ARs on the Sun. For two of them the flow patterns were investigated in terms of radiance and Doppler shifts of various emission lines that originate at different heights in the solar atmosphere. The flow patterns (blue and red shifts along the LOS of SUMER) are highly structured, and sharp spatial gradients (on the scale of arcseconds) were observed, which indicate the existence of sizable shear flows in ARs. The patterns vary with height (formation temperature of the line) but are fairly stable at large scales (of the order of the associated sunspot), indicating quasi-steady flows in the corona in ARs.

One active region was scanned back and forth (from east to west and return) to detect temporal changes. This procedure yielded Dopplermaps which were found to be stable at mesoscopic scales but varied substantially at smaller scales (arcseconds). This result hints at small-scale plasma dynamics in the AR. But it also indicates the steadiness of the global flow pattern, which may correspond to continuous large-scale plasma convection in the active corona. Usually, spectroscopic observations are hampered by the lack of magnetic field measurements, and suffer from projection effects of the emission when seen without altitude reference against the disk. Therefore, we studied for the first time an AR in close connection with its 3-D magnetic field, as extrapolated from simultaneous measurements of the underlying magnetic field in the photosphere.

Such force-free extrapolations based on photospheric magnetograms allowed us to correlate in detail the field topology of one AR with its radiance and Dopplershift pattern, which in turn permits a reconstruction of the three-dimensional distribution and flow of the plasma in the corona. Here the emission of neon was used as a valuable tracer ion of the bulk material. This comparison revealed the complex association on all scales of plasma and field, for example in open or closed topologies. The weak shearing of the calculated field in the AR implies fieldaligned currents to flow everywhere, and often field-topology changes are found to coincide with sharp changes in the flows, e.g. from up to down along the LOS and vice versa.

Although this study was intended to be mainly phenomenological, we finally also derived empirical values for the coronal mass flux in loops and of the coronal current density that is consistent with the calculated $\alpha$, which is a quantitative measure for non-potentiality. Lack of independent plasma density diagnostics did not enable us to derive for our data set the plasma density and the mass flux quantitatively.

This study clearly illustrates the future need for simultaneous measurements on all scales of the (vector) magnetic field and of the plasma by means of imaging and spectroscopy. Only through such a combined effort can further progress be expected in understanding the dynamics and energetics of the active solar corona.

Acknowledgements. The SUMER project is financially supported by DLR, CNES, NASA and the ESA PRODEX programme (Swiss contribution). SUMER and MDI are part of SOHO, the Solar and Heliospheric Observatory of ESA and NASA. We thank the MDI team for the magnetic field data. NSO/Kitt Peak data used here are produced cooperatively by NSF/NOAO, NASA/GSFC, and NOAA/SEL. We thank the NSO/KP for the use of their magnetic field data. Some of the images were taken from the SUMER homepage. They were processed and provided by I. E. Dammasch, whom we therefore want to thank. The work of Wiegelmann was supported by the DLRgrant 50 OC 0007. 


\section{References}

Amari, T., Aly, J. J., Luciani, J. F., Boulmezaoud, T. Z., \& Mikic, Z. 1997, Sol. Phys., 174, 129

Amari, T., Boulmezaoud, T. Z., \& Mikic, Z. 1999, A\&A, 350, 1051

Antonucci, E. 1994, Space Sci. Rev., 70, 149

Aschwanden, M. J. 2004, Physics of the Solar Corona - An Introduction (Chichester UK: Praxis Publishing Ltd., International Publishers in Science and Technology), and (Berlin: Springer), http://www.lmsal.com/ aschwand/eprints/2002-praxis/

Aschwanden, M. J., Poland, A. I., \& Rabin, D. M. 2001, ARA\&A, 39, 175

Aschwanden, M. J., De Pontieu, B., Shrijver, C. J., \& Title, A. 2002, Sol. Phys., 206, 99

Aschwanden, M. J., Newmark, J. S., Delaboudiniere, J.-P., et al. 1999, ApJ, 515, 842

Brekke, P. 1998, Three-dimensional Structure of Solar Active Regions, in Second Advances in Solar Physics Euroconference, ed. C. E. Alissandrakis, \& B. Schmieder, ASP Conf. Ser., 155, 150

Carcedo, L., Brown, D. S., Hood, A. W., Neukirch, T., \& Wiegelmann, T. 2003, Sol. Phys., 218, 29

Charbonneau, P. 1995, ApJS, 101, 309

Chiu, Y. T., \& Hilton, H. H. 1977, ApJ, 212, 821

Dammasch, I. E., Wilhelm, K., Curdt, W., \& Hassler, D. M. 1999, A\&A, 346, 285

de Pontieu, B. 2003, TRACE and Ground-based Observations of Small-scale Dynamics in Active Regions, in Turbulence, Waves and Instabilities in the Solar Plasma, ed. R. Erdelyi, K. Petrovay, B. Roberts, \& M. Aschwanden (Dordrecht: Kluwer Academic Publishers), 277

Gurman, J. B., \& Athay, R. G. 1983, ApJ, 273, 374

Harrison, R. A., Fludra, A., Pike, C. D., et al. 1997, Sol. Phys., 170, 123

Harvey, K. L., \& Harvey, J. W. 1976, Sol. Phys., 47, 233

Hassler, D. M., Dammasch, I. E., Lemaire, P., et al. 1999, Science, 283, 810

Jones, H. P., Duvall, Jr. T. L., Harvey, J. W., et al. 1992, Sol. Phys., 139, 211

Keil, S. L., Balasubramaniam, K. S., Bernasconi, P., Smaldone, L. A., \& Cauzzi, G. 1994, Solar active region evolution: comparing models with observations, 14th International Summer Workshop, National Solar Observatory / Sacramento Peak, ASP Conf. Ser., 68, 265

Kjeldseth-Moe, O. 2003, The Solar Transition Region, in Dynamic Sun, ed. B. N. Dwivedi (Cambridge University Press), 196

Lagg, A., Woch, J., Krupp, N., \& Solanki, S. K. 2004, A\&A, 414, 1109

Lemaire, P., Wilhelm, K., Curdt, W., et al. 1997, Sol. Phys., 170, 105

Liewer, C. P., Neugebauer, M., \& Zurbuchen, T., in Solar Wind Ten, ed. M. Velli, R. Bruno, \& F. Malara, AIP Conf. Proc., 679, Melville, NewYork, USA, 51, 2003

Meunier, N., \& Kosovichev, A. 2003, A\&A, 412, 541

Moses, D., Clette, F., Delaboudiniere, J.-P., et al. 1997, Sol. Phys., 175,571
Neugebauer, M. 2001, The solar-wind and heliospheric magnetic field in three dimensions, in The Heliosphere Near Solar Minimum The Ulysses Perspective, ed. A. Balogh, R. G. Marsden, \& E. J. Smith (Berlin: Springer-Verlag), 43

Patsourakos, S., Klimchuk, J. A., \& MacNeice, P. J. 2004, ApJ, 603, 322

Peter, H. 2001, A\&A, 374, 1108

Pevtsov, A. A., Canfield, R. C., \& McClymont, A. N. 1997, ApJ, 481, 973

Qiu, J., Wang, H., Chae, J., \& Goode, P. R. 1999, Sol. Phys., 190, 153

Rosner, R., Tucker, W. H., \& Vaiana, G. S. 1978, ApJ, 220, 643

Roumeliotis, G. 1996, ApJ, 473, 1095

Sakurai, T. 1981, Sol. Phys., 69, 343

Serio, S., Peres, G., Vaiana, G. S., Golub, L., \& Rosner, R. 1981, ApJ, 243, 288

Scherrer, P. H., Bogart, R. S., Bush, R. I., et al. 1995, Sol. Phys., 162, 129

Schmidt, H. V. 1964, in ASS-NASA Symposium on the Physics of Solar Flares, ed. W. N. Ness, NASA SP-50, 107

Seehafer, N. 1978, Sol. Phys., 58, 215

Semel, M. 1967, Ann. Astrophys., 30, 513

Semel, M. 1988, A\&A, 198, 293

Solanki, S. K., Lagg, A., Woch, J., Krupp, N., \& Collados, M. 2003, Nature, 425, 692

Spadaro, D., Lanzafame, A. C., Consoli, L., et al. 2000, A\&A, 359, 716

Teriaca, L., Banerjee, D., \& Doyle, J. G. 1999, A\&A, 349, 636

van Driel-Gesztelyi, L. 2003, Observational Signatures of Magnetic Reconnection, in Turbulence, Waves and Instabilities in the Solar Plasma, ed. R. Erdelyi, K. Petrovay, B. Roberts, \& M. Aschwanden (Dordrecht: Kluwer Academic Publishers), 297

Wheatland, M. S., Sturrock, P. A., \& Roumeliotis, G. 2000, ApJ, 540, 1150

Wiegelmann, T., \& Neukirch, T. 2002, Sol. Phys., 208, 233

Wiegelmann, T., \& Neukirch, T. 2003, Nonl. Proc. Geophys., 10, 313

Wiegelmann, T. 2004, Sol. Phys., 219, 87

Wilhelm, K., Curdt, W., Marsch, E., et al. 1995, Sol. Phys., 162, 189

Wilhelm, K., Lemaire, P., Curdt, W., et al. 1997, Sol. Phys., 170, 75

Wilhelm, K., Dammasch, I. E., Marsch, E., \& Hassler, D. M. 2000, A\&A, 353, 749

Wilhelm, K., Dwivedi, B. N., Marsch, E., \& Feldman, U. 2004, Space. Sci. Rev., 111, 415

Winebarger, A. R., DeLuca, E. E., \& Golub, L. 2001, ApJ, 553, L81

Winebarger, A. R., Warren, H., van Ballegooijen, A., DeLuca, E. E., \& Golub, L. 2002, ApJ, 567, L89

Xia, L. D. 2003, Ph.D. Thesis, Georg-August-Universität, Göttingen

Xia, L. D., Marsch, E., \& Curdt, W. 2003, A\&A, 399, L5

Xia, L. D., \& Marsch, E. 2003, in Solar Wind Ten, ed. M. Velli, R. Bruno, \& F. Malara, AIP Conf. Proc., 679, 319

Yoshimura, H., Tanaka, K., Shimizu, M., \& Hiei, E. 1971, PASJ, 23, 443 